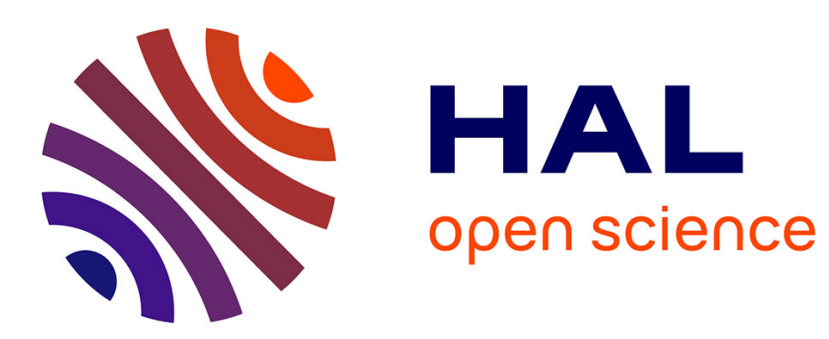

\title{
Saving Face and Group Identity
}

Tor Eriksson, Lei Mao, Marie Claire Villeval

\section{To cite this version:}

Tor Eriksson, Lei Mao, Marie Claire Villeval. Saving Face and Group Identity. Experimental Economics, 2017, 20 (3), pp. 622-647. 10.1007/s10683-016-9502-3 . halshs-01393311

\section{HAL Id: halshs-01393311 https://shs.hal.science/halshs-01393311}

Submitted on 10 Jul 2019

HAL is a multi-disciplinary open access archive for the deposit and dissemination of scientific research documents, whether they are published or not. The documents may come from teaching and research institutions in France or abroad, or from public or private research centers.
L'archive ouverte pluridisciplinaire HAL, est destinée au dépôt et à la diffusion de documents scientifiques de niveau recherche, publiés ou non, émanant des établissements d'enseignement et de recherche français ou étrangers, des laboratoires publics ou privés. 


\title{
Published in Experimental Economics 2017
}

\section{Saving Face and Group Identity}

\author{
Tor Eriksson, Lei Mao and Marie Claire Villeval*
}

\begin{abstract}
Are people willing to sacrifice resources to save one's and others' face? In a laboratory experiment, we study whether individuals forego resources to avoid the public exposure of the least performer in their group. We show that a majority of individuals are willing to pay to preserve not only their self- but also other group members' image, even when group identity is minimal. When group identity is made more salient, individuals help regardless of whether the least performer is an in-group or an out-group. In contrast, people are less likely to sacrifice for individual strangers, showing a major role for group identity and reputation concerns within groups relative to an interpretation in terms of moral norms.
\end{abstract}

JEL-Code: C92, D03, M52, Z13

Keywords: Saving face, social image, pro-social behavior, group identity, experiment.

\section{Contact Information:}

* Corresponding author

Tor Eriksson, Department of Economics and Business, Aarhus School of Business and Social Sciences, Aarhus University. Fuglesangs Allé 4, 8210 Aarhus V, Denmark. E-mail: tor@asb.dk.

Lei Mao, School of Insurance, Central University of Finance and Economics, Beijing, China. E-mail: maolei3518@sina.com.

Marie Claire Villeval, Univ Lyon, CNRS, GATE UMR5824, 93, Chemin des Mouilles, F-69130 Ecully, France; IZA, Bonn, Germany; Department of Public Finance, University of Innsbruck. E-mail:

villeval@gate.cnrs.fr.

Acknowledgments: We are grateful to participants at the EALE-SOLE World Congress in Montreal, the Economic Science Association in New-York, the $3^{\text {rd }}$ Annual Xiamen University International Workshop on Experimental Economics, the ASFEE conference in Montpellier, the workshop on Cooperation, cultural aspects and norms in Jerusalem, and seminar participants at GATE for valuable comments on an earlier version of this paper. We also address special thanks to F. Galeotti and D. Houser for very useful comments. We thank S. Ferriol, R. Cautain and Q. Thévenet for programming this experiment and T. He and Y. Chen for excellent research assistance. This research program has been supported by a grant from the French National Research Agency (ANR, EMCO program, HEIDI grant) and was performed within the framework of the LABEX CORTEX (ANR-11-LABX-0042) of Universite de Lyon, within the program "Investissements d'Avenir" (ANR-11-IDEX-007) operated by the French National Research Agency (ANR). 


\section{INTRODUCTION}

The concern for one's and other's image is crucial for diplomacy, informal contract enforcement, maintenance of business networks, good employment relationships, successful bargaining situations (Ting-Toomey, 2005). Yet, individuals or organizations' image may be threatened by the development of practices like the dissemination of performance ratings that praise the best performers but may also shame the least performers. Examples include citation indices and rankings of researchers in academia, ranking of sellers on on-line purchasing websites, employee performance appraisals in organizations. ${ }^{1}$ The previous literature has focused on the implications of individual rankings on performance and efficiency, showing mixed results: While rankings motivate some individuals to outperform others, they may also discourage less able individuals. But another source of utility loss has not received much attention in the economic literature: rankings may entail losses of utility in terms of image that people may strive to avoid, and this is precisely what we are studying in this paper.

If people make efforts to protect their own image, are they also willing to sacrifice monetary resources to save other's face? Social psychologists have shown how preserving image is important for humans in society (Spencer et al., 2001; Baumeister et al., 2005; Mruk, 2006). Sociologists have studied how self-esteem and considerateness for others lead people to preserve self- and others' face in social encounters. Goffman has defined face as “an image of self delineated in terms of approved social attributes" $(2005$, p.5), and shown that face-saving practices are a condition for social interactions. In contrast, economists have not widely investigated the importance of face-saving for the self and others. ${ }^{2}$ This study aims at contributing to understand face saving by combining the economic analysis of image and social preferences. Recent theories (Benabou and Tirole, 2006; Koszegi, 2006) and empirical tests of image concern (Johansson-Stenman and Svedsater, 2012; Johansson-Stenman and Martinsson,

\footnotetext{
1 Many firms struggle to find a well-functioning performance evaluation system. Recently, well-known companies have abolished their annual performance review (Adobe) or moved from a rigid, forced ranking approach to systems that are more flexible (Microsoft, Yahoo). One aspect that is not recognized in the literature is the loss of face (selfas well as others' image) associated with performance evaluations in which individuals' ranks are publicly exposed. ${ }_{2}$ An exception is Hugh-Johns and Reinstein (2010) who study loss of face as the disutility for common-knowledge of rejection. The fear of losing face leads to inefficiencies in markets because it leads to fewer offers.
} 
2005; Alpizar et al., 2008; Ariely et al., 2009; Lacetera and Macis, 2010; Cappelen et al., 2012) demonstrate that individuals care about how others perceive their actions. People are motivated by what others think about them (Ellingsen and Johannesson, 2007, 2008a; Eriksson and Villeval, 2011). These studies do not, however, directly examine the value for individuals to maintain selfand others' face.

In this paper, we study experimentally whether people sacrifice monetary resources to avoid being publicly exposed - i.e. to save face - when exposure signals that the individual's performance is the lowest compared to others. Another original feature is analyzing how large is the share of individuals who accept incurring costs to save the face of others without reaping any personal material benefit from this action. Testing face saving would be very difficult by means of survey or registry data. Therefore, we have designed a laboratory experiment that we tested in China from where the concept of face (mien-tzu) originates (Ho, 1976). ${ }^{3}$ In the Minimal (group) Identity treatment subjects are matched in triads throughout the session and they have to perform a real-effort task for a fixed payment. In one part, those whose performance is lowest in their triad are singled out and briefly publicly exposed. Although the equilibrium is not exerting any effort, public exposure may be perceived as signaling a lower status. It is thus susceptible to shame individuals. ${ }^{4}$ It can however be avoided if at least two triad members are willing to pay a fee. This one-shot decision allows us to measure the likelihood of sacrificing resources to save one's and others' image.

Our first contribution is showing that most individuals strive to save face and agree to sacrifice resources to avoid public exposure, and that more than half of the individuals also sacrifice to avoid others' exposure. Such a behavior can be related to empathy, altruism (Andreoni and Miller, 2002) and norm enforcement to maintain the integrity of a society (Fehr and Fischbacher, 2003) in the domain of image. We show that altruistic behavior extends beyond

\footnotetext{
${ }^{3}$ Face is a key dimension in the Confucian culture to orchestrate social interactions (Hu, 1944; Redding and Ng, 1982; Yang, 1989; Qi, 2011). The concern of Chinese for face results from a socialization process using shaming techniques to inculcate strong sensitivity to group belonging and others' opinion (Redding and Ng, 2002). The concern for others' image is called "giving face" in Chinese.

${ }^{4}$ We kept the procedure as soft as possible to avoid creating too much embarrassment. In psychology, Smith et al. (2002) have shown that shame has two core features: its links with public exposure and with negative self-evaluation.
} 
the pecuniary realm and includes taking actions that benefit the social standing of others.

Then, we investigate whether the willingness to avoid others' exposure is sensitive to the saliency of group identity. Indeed, the link between identity, on the one hand, and face, on the other hand, has been little explored in the literature (Spencer-Oatey, 2007). Therefore, we study whether individuals are more willing to pay for saving the face of group members rather than the face of strangers, and the face of in-groups than the face of out-groups. By addressing these questions, we contribute to the recent economic literature on group identity (Akerlof and Kranton, 2000; Bowles and Gintis, 2004; Fershtman and Gneezy, 2001; Bernhard et al., 2006; Charness et al., 2007; Chen and Li, 2009; Chen and Chen, 2011; Hargreaves Heap and Zizzo, 2009; Zizzo, 2011; Goette et al., 2012; Masella et al., 2014).

While in the Minimal Identity treatment triad membership induces a minimal group identity, in other treatments we vary the strength of group identity. We manipulate the social distance in several directions, either reducing it by adding a "label identity" to triad members, or increasing it by withdrawing the notion of triads. We enhanced group identity in the same spirit as Chen and Chen (2011) in their study of the role of identity in the selection of equilibria in coordination games. To reinforce the prominence of group membership we gave painter names to groups, like in the method developed in social psychology by Tajfel et al. (1971) and Tajfel and Turner (1979), and like Chen and Chen (2011) we added an opportunity for the players to communicate during the identity formation stage. In the Homogenous (label) treatment, the triad members share the same label (Klee or Kandinsky, as explained below), while in the Heterogeneous (label) treatment labels are mixed within each triad. In these two treatments, triads remain fixed throughout the session. In contrast, in the No (group) Identity treatment we no longer mention the notion of triad and individuals are matched with two randomly rematched participants at the beginning of each new part. In one part individuals can still pay for avoiding the exposure of the least performer among themselves and two other co-participants but in contrast with the Minimal Identity treatment these co-participants are strangers. This removes any potential reputation concerns. When people hold the same label the social distance should be reduced, while it should 
increase when they do not, compared to the Minimal Identity treatment. These treatments allow us to identify whether there is a conflict between a general norm of saving others' face and another norm according to which one should save the face of in-groups but not of out-groups. We can thus measure how image management is affected by group membership.

Our second contribution is showing that, compared to the Minimal Identity treatment, strengthening group identity or creating heterogeneous groups makes no significant difference with respect to the choice to save one's and other's face. People are willing to help out-group as much as in-group members in their triad. The analysis of communication during the identity formation stage indicates that asking and receiving help during this stage does not affect significantly the decision to save others' face in any treatment during the game. In the No Identity treatment, more than one third of the individuals are still willing to help to save a stranger's face but this proportion is significantly lower than in the other treatments. These findings show a major role of the triad identity relative to an interpretation in terms of moral norms which would have required finding a similar proportion of sacrifices across treatments. This lower empathy for strangers may be driven by a higher social distance with others when there is no group identity and by the absence of reputation concerns (although when triads are fixed, players are also unaware of the content of the next part when they decide to pay the fee for avoiding exposure). The absence of a correlation between receiving help during the identity building part and helping others in the game suggests that general reciprocity is not driving the difference between the identity and the no identity treatments.

Finally, we examine how public exposure affects effort. Ex ante, the threat on image may either crowd-out intrinsic motivation or boost performance to avoid exposure. For example, taxpayers are more compliant when detection of fraud leads to the public display of the evaders' pictures (Coricelli et al., 2010). Ex post, the shaming effect of public exposure may also either crowd-in intrinsic motivation if individuals work harder to restore their image, or crowd-out it if image has been altered (Coricelli et al., 2014). We carry out performance comparisons between each treatment and a No Feedback treatment in which subjects do not receive any social 
information and have no risk of public exposure. This additional treatment allows us to disentangle the effect of learning across parts and the effect of social information and exposure on performance. We fail to find evidence of an ex ante effect of the threat of exposure on performance, but we establish some evidence that having been exposed tends to reduce immediate future performance at both the individual and the triad levels. In contrast to Masella $e t$ al. (2014) who found that identity modulates the impact of incentives schemes on performance, we find no effect of group identity on the impact of exposure on performance.

Overall, these findings have implications for the importance of protecting people's face and for firms to avoid modes of evaluation or feedback policies that may threaten the social image of individuals in the organization or the society.

In the remainder of the paper, Section 2 summarizes the related literature. Section 3 describes the experimental design and procedures and presents our behavioral conjectures. Section 4 reports our findings and Section 5 discusses the results and concludes.

\section{RELATED LITERATURE}

Our paper contributes to three literatures. One is studies of identification and audience effects. These studies have shown that prosocial choices are affected by identification (Bohnet and Frey, 1999; Charness and Gneezy, 2008) and anticipated verbal feedback (Ellingsen and Johannesson, 2008b), and that punishment in public promotes norm obedience (Xiao and Houser, 2011). While these studies ensure the anonymity of interactions, our design exposes singled-out individuals to an audience. The presence of a non-anonymous audience has been shown to increase prosociality (Ariely et al., 2009), tax compliance (Coricelli et al., 2010), coordination on the audience's preferred outcome in the battle-of-sexes game and defection in the prisoner's dilemma (Charness et al., 2007), and inflation of stated self-assessment (Ewers and Zimmerman, 2015). These studies point to the importance of social approval. ${ }^{5}$ Our analysis complements them by

\footnotetext{
5 There is also a literature on the willingness-to-pay for privacy, showing that people are less willing to disclose personal information when information is more sensitive (Feri et al., 2013), or in the absence of economic advantage associated with disclosure (Beresford et al., 2012). While we are also concerned with the privacy of sensitive data, we differ from this literature in which disclosure is voluntary.
} 
examining whether people sacrifice resources to preserve image.

Second, we contribute to the literature on group identity. In naturally occurring groups, in-group favoritism has been observed in norm enforcement (Bernhard et al. (2006) because of social ties (Goette et al., 2006, 2012a). Discrimination of the opposite group has been identified both in dictator games (Ben-Ner et al., 2009; Abbink and Harris, 2012) and competition games (Goette et al., 2012b; Kato and Shu, 2013). Using the minimal group paradigm, studies have shown that group identity strengthens prosocial behavior (Chen and $\mathrm{Li}, 2009$ ) while people act more selfishly when opposed to out-groups (Kollock, 1998; Charness et al., 2007; Zizzo, 2011), and that trust decreases with group membership because of discrimination against out-groups (Hargreaves Heap and Zizzo, 2009). ${ }^{6}$ It has also been shown that making group identity more salient can lead to in-group coordination to the efficient equilibrium in a minimum effort game (Chen and Chen, 2011). Our contribution is a study of whether face saving is a general norm or a discriminatory process conditional on the social distance with the victim. We also contribute to the analysis of the impact of the group identity saliency on this type of pro-social behavior.

Third, we contribute to the literature on the incentive effects of social status. Status can be conferred by public recognition, performance feedback and ranking. These measures act as incentives on effort (Kosfeld and Neckerman, 2011; Kuhnen and Tymula, 2012; Tran and Zeckhauser, 2012; Bradler et al., 2013) and cooperation (Eckel et al., 2010), although their effect is not unequivocal (Barankay, 2012; Charness et al., 2014). By singling-out the least productive individuals we can examine the impact of anticipated decreased status on further performance. ${ }^{7}$

\section{EXPERIMENTAL DESIGN AND PROCEDURES}

\subsection{Treatments}

The experiment consists of four main treatments: a Minimal Identity treatment and three

\footnotetext{
6 This is not systematic, however, as the impact of group identity on behavior is conditional on the saliency of identity (Eckel and Grossman, 2005), the procedure used to generate identity (Guala et al., 2013), the group size (Harris et al., 2009), the mode of group formation (Herbst et al., 2012), the existence of inter-group conflicts (Chakravarty and Fonseca, 2011), and culture (Buchan et al., 2006).

By means of a trust game, Galeotti and Zizzo (2014) analyze the impact of singling-out individuals depending on whether it is random or it results from people's preferences. Singling-out individuals reduces trustworthiness and majority group members discriminate against singled-out subjects.
} 
treatments in which we either remove or increase the saliency of group identity. A final treatment has been added to control for learning and the evolution of performance over time.

\section{Minimal Identity treatment}

Each session consists of four parts and players are informed about the content of a part only at the beginning of each part (see instructions in Appendices 1 and 2). At the beginning of the first part, we randomly form anonymous triads that remain fixed for the entire session, and this is common knowledge. In each part, subjects have to perform a simple task during four minutes. More precisely, they have to pick an apple on the screen and move it into a collecting basket located at the bottom of the screen. As soon as an apple enters the basket, a new apple appears at a random spot on the screen. Each apple moved into the basket earns one point. A counter displays the current score. The task does not require any skill and the score is a proxy of the level of effort. ${ }^{8}$ Subjects receive a fixed wage of 100 ECU (Yuan 10, about U.S. \$1.65) in each of the four parts.

Parts differ from each other in terms of feedback and risk of exposure. At the end of part 1, subjects learn their own score. At the end of part 2, they are also informed about the score and the rank of each member in their triad, with rank 1 assigned to the best performer and rank 3 to the least performer. In case of ties, ranks are assigned randomly between the ties. At the end of part 3, they receive the same type of feedback about their performance in part 3. Moreover, subjects with rank 3 based on their relative performance in part 3 are requested to move to the front of the room one by one before coming back to their seat. These rules are made common information in the instructions. Public exposure can, however, be waived if at least two triad members choose to pay to avoid the public exposure of the member with rank 3. ${ }^{9}$ In this case, 10 ECU are deducted from the payoff of those who chose to pay. If less than two triad members chose to pay the fee, the member with rank 3 is publicly exposed and no deduction is made. Each member is informed

\footnotetext{
${ }^{8}$ Using a task in which a low performance would signal low cognitive ability would have generated more embarrassment in case of exposure, which we wanted to avoid for ethical reasons. Thus, the intensity of shame due to public exposure in a professional or educational environment is probably underestimated in our experiment. 9 This raises a coordination issue in the group that will be studied later. To avoid this coordination problem, an alternative design could have been to randomly choose one of the triad members' decisions to determine the exposure of the least performer. However, uncertainty of implementation would have limited the feeling of responsibility and probably reduced the willingness to pay for avoiding exposure.
} 
about each triad member's decision whether to offer to pay or not. Finally, part 4 replicates the features of part 2: after performing the task again during four minutes, subjects are informed on the score and rank of each triad member based on their relative performance in part 4. But in contrast to part 3 , there is no risk of exposure. ${ }^{10}$ Since subjects discover the content of each part sequentially, they cannot adjust their effort strategically in part 2 to manipulate others' beliefs about the effort in the triad in part 3.

We elicited the emotional state of the subjects by using a self-reported emotion survey as in Hopfensitz and Reuben (2009). Although we are chiefly interested in the feelings of shame and happiness, at several steps ${ }^{11}$ subjects report the intensity of ten emotions that they may experience at this precise moment (anger, contempt, shame, envy, sadness, happiness, guilt, gratefulness, fear, relief) on a Likert-type scale from 1 to 7 (the highest possible intensity).

\section{Homogeneous/Heterogeneous Label treatments}

In the Minimal Identity treatment, belonging to a triad may generate a group identity. To explore whether the willingness to save others' face is affected by the social distance between members, we have designed two conditions of a treatment in which we add another group affiliation that is congruent or not with the triad membership. In the homogeneous label condition (hereafter, "Homogenous treatment"), triads are formed of subjects who received the same label (Klee or Kandinsky). ${ }^{12}$ Thus, group identity deriving from triad membership is reinforced and social distance is further reduced. In the heterogeneous label condition (hereafter, "Heterogeneous treatment"), triads are formed of subjects who received different labels. Thus, compared to the Minimal Identity treatment, social distance may be increased in the triad if the least performer has a different label than the other triad members.

\footnotetext{
10 We acknowledge that the impact of exposure on image cannot be disentangled precisely from that of lifting anonymity or the displeasure to stand in front of the public. But if the decision to sacrifice was motivated by the lift of anonymity or the displeasure of exposure and not because of image concerns, then it should be independent of the rank of the exposed player. We will show later that this is not what we observe, thanks to an additional treatment similar to the Baseline, except that it is the best performer who is publicly exposed unless at least one player pays to avoid exposure $(\mathrm{N}=36)$.

That is at the beginning of the session, after receiving feedback at the end of part 1 , at the end of part 2, during part 3 after choosing to pay to avoid public exposure, after the exposure stage, and at the end of part 4.

12 Note that, as far as we know, we are the first to use these Klee-Kandinsky labels in China.
} 
Labels are assigned in a preliminary part for the whole session, using a procedure inspired by the minimal group paradigm of Tajfel (1971). This part consists of two stages. In the first stage, subjects are randomly assigned to one of two groups of similar size, named the Kandinsky group and the Klee group, respectively. In the second stage, they engage in a recognition task. They review five pairs of paintings created by Klee and Kandinsky respectively, during 20 seconds each. The screen indicates which artist painted each painting. Next, they review two anonymous paintings successively and they are requested to report which artist painted each of them (Klee or Kandinsky). Each correct answer pays 50 ECU. Before entering their individual responses, like in Chen and Chen (2011) subjects have the opportunity to communicate with their in-groups by means of a chat box to try to solve the problems. They can chat during three minutes. They receive a feedback on the correctness of answers at the end of the session. ${ }^{13}$

Once labels have been assigned, the rest of the experiment is similar to the Minimal Identity treatment except that individuals are aware of the composition of their triad in terms of group identity and that publicly exposed individuals have to indicate aloud their label. Like in the Minimal Identity treatment, triad members are identified by their ranking and their score, as well as whether they are members of the Klee or Kandinsky group, but the group members remain anonymous.

\section{No Identity treatment}

In the No Identity treatment, subjects are not working in a triad. In each part starting after part 1, we compare their performance with the performance of two other randomly selected subjects in the same session, and we rematch players randomly in each new part. Subjects are asked whether they are willing to pay for avoiding the exposure of the least performer among the three subjects who are matched together in part 3 , knowing that these subjects are likely not the same as in the previous part. Therefore, the social distance with the potentially exposed least performer is higher

\footnotetext{
13 To check whether the group inducement mechanism was effective, subjects had to report their feeling intensity of belonging to their group, with 1 referring to no feeling of group membership at all and 7 referring to a very strong feeling of group membership. The average reported number is 5.03, which indicates that the mechanism worked.
} 
compared to the Minimal Identity treatment and since players are strangers, there is no possible reputation concern. With these manipulations, we can measure whether the decision to sacrifice to save face is dependent on social distance and reputation.

\section{No Feedback treatment}

Comparisons of performance in parts 1 and 2 reveal whether individuals adjust effort when they anticipate being informed about their relative performance. Comparing performance in parts 2 and 3 indicates whether the perspective of being publicly exposed boosts effort or crowds-out intrinsic motivation. Finally, comparing performance in parts 3 and 4 measures whether exposure has a detrimental effect on effort in part 4. However, this interpretation is conditional on people not learning the task over time. To control for learning across parts, we have run a control treatment. This treatment includes the same number of parts and players are matched in triads as in the other treatments. But in contrast to the other treatments, there is no risk of public exposure and participants do not receive any feedback on any of the other's performance in any part. The only information they get at the end of each part is their own score. The comparison between this treatment and the Minimal Identity treatment helps us to disentangle the impact of learning and the impact of social information and exposure on performance.

\section{Belief elicitation}

Requiring that two out of three triad members pay the fee to avoid exposure may give rise to a coordination problem. We have therefore elicited the participants' beliefs about the number of other triad members who will choose to pay the fee $(0,1$ or 2$)$. This question was asked just after the individuals made their decision in part 3 and it was not mentioned in the instructions to avoid focusing attention on the coordination issue. A correct prediction paid 50 ECU. This question has been introduced after the first eight sessions.

\subsection{Procedures}

The experiment was conducted at the experimental laboratory of Beijing Normal University, Beijing, China. Posters inviting students to participate were posted on online campus forums of 
BNU and Beijing University of Posts and Telecommunications and flyers were distributed on the campuses. In total 420 students from various disciplines participated in 24 sessions with 18 participants in each (12 only in the sessions with the No Feedback treatment). Table 1 reports summary statistics on sessions. The proportion of female participants exceeds $50 \%$ in all treatments (binomial test, $p<0.001$ ); thus we control for a possible gender effect in the regression analysis.

\section{(Insert Table 1 about here)}

The experiment was computerized using the REGATE software. Upon arrival, subjects were randomly assigned to a computer. Talking was not allowed. Instructions were distributed and read aloud after completion of each part. We checked that each subject understood correctly the instructions and all questions were answered in private. Before the beginning of the first part, subjects were allowed to practice the task during two minutes to minimize learning effects during the rest of the session. Each session lasted about 90 minutes, including a final demographic survey and payment in private. Subjects earned on average 45 Yuan (\$7.38), which corresponds to approximately a 90 minute-wage for a part-time job in Beijing.

\subsection{Behavioral conjectures}

Predictions based on standard theory with selfish preferences and purely extrinsic motivation are straightforward. Since individuals are paid a fixed wage, they should exert no effort in any part. Individuals should not pay to avoid exposure and the threat of exposure should not affect effort since exposure does not impact payoffs. These predictions hold for all treatments and conditions.

However, instead of maximizing their monetary payoff, individuals may try to maximize their utility that depends on several sources of motivation. In particular, as in Benabou and Tirole (2006), an individual's utility may depend on extrinsic motivation (how much he values money), intrinsic motivation (how much he enjoys performing the task), and image motivation (how much he cares about his own and other's exposure). Utility is affected negatively by the variable cost of providing effort and the fixed cost of preserving image if the individual actually pays the fee. 
Image motivation enters negatively in the utility function if one assumes that public exposure entails a loss of image. An individual with image concern will suffer a loss if he is exposed or if somebody else is exposed, but this loss is expected to be higher when he experiences exposure himself. Of course, there should be no image utility loss if the individual has no image concern or if exposure is waived. Thus, when deciding whether or not to pay the fee, the individual compares the amount of this fee to the image loss he would suffer in case of public exposure, accounting for his belief about other triad members' decisions to pay the fee. Moreover, avoiding the loss of image should create an additional incentive to exert effort in part 3 for the triad members who learn that there is a risk of being exposed. Finally, actual exposure in part 3 may crowd-out the effort of the exposed triad members in part 4 if they feel they have lost face; this crowding-out effect is more unlikely for the other triad members since exposure means that they were not willing to pay to avoid this exposure.

We summarize our initial conjectures as follows:

Conjecture 1 (Sacrifice). Image-concerned individuals are more likely to pay the fee for avoiding their own exposure than for avoiding the exposure of another member.

Conjecture 2 (Effort). Ex ante, the cost of exposure in terms of image increases the individuals' level of effort when they learn that there is a risk of being exposed. Ex post, exposure crowds-out the intrinsic motivation of the least performer in the triad.

Manipulating group identity should have no impact on the willingness to pay the fee if saving others' face is a general social norm. Alternatively, the utility loss experienced may depend on the identity of the subject at risk. Indeed, compared to the Minimal Identity treatment the loss may be higher when the individual at risk is an in-group and lower when he is an out-group or a stranger. Manipulating group identity may also affect the beliefs about others' decision to pay the fee, increasing them when the least performer is an in-group, decreasing them when the least performer is an out-group or a stranger. It may help solving the coordination problem regarding who should pay the fee in heterogeneous triads, as an individual should be more likely to pay the fee when the least performer is an in-group and the other triad member an out-group. Additionally, effort may be affected by the composition of the group, except if saving 
face is a general social norm. Being matched with out-groups or strangers may boost performance to reduce the risk of being the least performer, as one may expect less help from others in these conditions.

We summarize the next conjectures as follows:

Conjecture 3 (Sacrifice and identity). The decision to pay the fee is more likely when the least performer belongs to the same triad or holds the same label than when he is an out-group or a stranger.

Conjecture 4 (Effort and identity). Being matched with out-groups in the Heterogeneous treatment boosts effort even further if individuals are more willing to report the risk of exposure on to out-groups.

\section{RESULTS}

We first examine the decision to pay for avoiding public exposure. Next, we focus on the emotions reported by the subjects and finally, we study efficiency as measured by effort levels.

\subsection{Avoidance of public exposure}

Table 2 displays summary statistics for the payment of the fee to avoid the public exposure of the least performer, by treatment and by performance rank in the triad in part 3 . The last column gives the percentage of least performers who are actually exposed.

\section{(Insert Table 2 about here)}

Table 2 shows that, on average, $75 \%$ of the least performers in the Minimal Identity treatment are willing to pay to avoid being publicly exposed. This percentage is $80.95 \%$ in the Homogeneous treatment, $71.43 \%$ in the Heterogeneous treatment, and $62.50 \%$ in the No Identity treatment. Pairwise proportion tests indicate that these values do not differ from the Minimal Identity treatment (two-sided, $p=0.569,0.754$, and 0.350 , respectively). This is confirmed by a Kruskal-Wallis test $(p=0.853)$. Thus, most subjects are willing to avoid exposure. ${ }^{14}$

\footnotetext{
${ }^{14}$ This is confirmed by the players' reports of their reasons for their decisions in the post-experimental survey. A majority responded that they chose to pay to avoid the least performers' losing face or feeling embarrassed.
} 
Table 2 also shows that in the Minimal Identity treatment a majority of subjects who are not personally at risk of public exposure (54.17\% of the best and the medium performers) choose to pay to waive the exposure of the least performer in their triad. This documents the importance of saving others' face. ${ }^{15}$ Interestingly, there is no significant difference between this percentage and the percentage in the Homogeneous treatment $(58.33 \%, p=0.642)$ and in the Heterogeneous treatment, regardless of whether the subject at risk is an in-group $(60.61 \%, p=0.565)$ or an out-group (52.94\%, $p=0.903)$ (pairwise comparisons, two-sided proportion tests). A Kruskal-Wallis test confirms that there are no significant differences between the Minimal Identity treatment and the other treatments $(p=0.862)$. To explain the lack of behavioral differences between the Homogeneous and the Heterogeneous treatments, we explored the content of communication during the group identity formation stage. A comparison of the percentages of subjects asking for help and receiving help from others is not significantly different in the Homogeneous treatment (28.57\%) and the Heterogeneous treatment (22.22\%) (proportion test with each painting group taken as an independent observation, $p=0.700$ ). Moreover, we find no significant difference either in the decision to pay the fee to save the least performer's face between the subjects who asked and got help during the group identity formation stage $(66.67 \%$ in the Homogeneous treatment and $71.43 \%$ in the Heterogeneous treatment, respectively) and those who did not (65.56\% and 58.16\%, respectively) (proportion tests, $p=0.905$ and 0.204 , respectively. This indicates that in our game general reciprocity is not the driver of helping behavior. ${ }^{16}$

Another important finding is that the percentage of subjects who are not at risk personally and who are willing to pay the fee in the No Identity treatment is $37.50 \%$. Two-sided proportion tests indicate that this differs from the Minimal Identity treatment $(p=0.101)$, the Homogeneous treatment $(p=0.021)$ and the Heterogeneous treatment when the least performer is an in-group

\footnotetext{
15 An alternative explanation is that the best and the medium performers' decision to pay is driven by potential shame if choosing not to pay since their decision is visible to the other triad members. However, the emotional data do not support this explanation: there is no significant difference in shame and happiness intensity between the best and the medium performers who chose to pay and those who made the opposite choice.

${ }^{16}$ Note that this is different from Chen and Chen (2011) who found that subjects asking more questions to their in-groups during the problem-solving task were more (less) cooperative with their in-(out-)groups in the main game.
} 
$(p=0.041)$. The percentage is significantly lower than when the least performer is an out-group but only if we consider a one-sided test $(p=0.065)$. A Kruskal-Wallis test indicates a significant difference between the No Identity treatment and the other treatments $(p=0.017)$. Although this percentage is lower than in most of the other treatments, it should still be considered remarkably high since in this treatment the social distance with the least performer is larger than in most other treatments. $^{17}$

As a consequence, the proportion of least performers who are actually publicly exposed varies from $28.57 \%$ in the Homogeneous treatment, $33.33 \%$ in the Heterogeneous treatment, $37.50 \%$ in the Minimal Identity treatment, to $66.67 \%$ in the No Identity treatment. Proportion tests indicate that there is no significant difference between the first three treatments $(p>0.100)$ while the No Identity treatment differs from the Minimal Identity treatment $(p=0.043)$, the Homogeneous treatment $(p=0.003)$ and the Heterogeneous treatment $(p=0.009)$.

In accordance with Conjecture 1, people are more likely to incur a cost for avoiding their own exposure than for waiving the exposure of another member. Indeed, tests confirm that the proportion of subjects who choose to pay the fee is higher among those who are at risk than among others (one-sided, $p=0.044$ in the Minimal Identity treatment, $p=0.006$ in the Homogenous treatment, $p=0.046$ in the Heterogeneous treatment, and $p=0.022$ in the No Identity treatment). The least performers hold also more pessimistic beliefs about the number of members who choose to pay. ${ }^{18}$

Table 3 reports an analysis of the determinants of the decision to pay the fee to prevent the public exposure of the least performer. It displays marginal effects from the estimates of five

\footnotetext{
17 The analysis of beliefs indicates, however, that the individuals are more pessimistic about others' willingness to pay the fee when they are matched with out-groups rather than with in-groups. The mean beliefs about the number of other triad members choosing to pay the fee are 1.36 (S.D.=0.68) in the Minimal Identity treatment, 1.42 (S.D.=0.73) in the Homogenous treatment, 1.11 (S.D.=0.74) in the Heterogeneous treatment, and $1.22($ S.D. $=0.75)$ in the No Identity treatment. Beliefs differ significantly between the Heterogeneous treatment on the one hand and the Minimal Identity treatment $(p=0.096)$ and the Homogenous treatment $(p=0.010)$ on the other hand (Mann-Whitney tests with each participant taken as one independent observation). They do not differ between the No Identity treatment and the other treatments $(p>0.100)$.

18 The mean beliefs of the least performers are 1.08 (S.D.=0.67) in the Minimal Identity treatment, 1.25 (S.D.=0.79) in the Homogenous treatment, 0.87 (S.D.=0.74) in the Heterogeneous treatment, and 0.83 (S.D.=0.76) in the No Identity treatment. They are significantly more pessimistic than others in the Minimal Identity treatment $(p=0.070)$, Heterogeneous $(p=0.057)$ and No Identity treatments $(p=0.002)$ (Mann-Whitney tests), not in the Homogeneous treatment $(p=0.185)$.
} 
Probit models. In model (1), the independent variables include a dummy variable for each treatment, with the Minimal Identity treatment taken as the reference category. They include a dummy variable for the sessions in which we elicited beliefs on the number of triad members willing to pay the fee and two dummy variables indicating whether the subject has got the first or the third rank, respectively, with the second rank taken as the reference. Model (2) augments model (1) with two variables capturing the beliefs that one or two other triad members choose to pay the fee (compared to none) interacted with the dummy variable for the belief elicitation condition. The aim is to test whether subjects try to solve a coordination problem, i.e. whether they are more (less) likely to pay the fee when they believe that one (two) other triad member will pay. Model (3) re-estimates model (2) on the sub-sample of the least performers. We include an additional dummy variable indicating whether the least performer is matched with two out-groups in the Heterogeneous treatment. Model (4) re-estimates model (2) on the sub-sample of the best and medium performers, after including a dummy variable that indicates whether the least performer is an out-group in the Heterogeneous treatment. Finally, model (5) restricts the observations to the best and medium performers in the Heterogeneous treatment. It tests the hypothesis that the subject's willingness to sacrifice is reduced when being matched with two out-groups and when the least performer is the only out-group in the triad. The reference category is the case in which the least performer is the subject's in-group. It also controls for the belief elicitation condition and the belief on the number of other triad members willing to pay the fee. ${ }^{19}$ In the five models, we control for demographic variables, including gender, age and family relative wealth. As these variables were never significant, we omit reporting their marginal effects. Standard errors are clustered at the triad level, except in model (3) since here we only consider one player per triad.

\section{(Insert Table 3 about here)}

\footnotetext{
19 This last variable replaces the separate belief variables included in the previous models because there is not enough variation in the decision to pay the fee when the subjects believe that the two other members will pay. $52.78 \%$ of subjects with rank 1 or 2 are willing to pay in the condition where we do not elicit beliefs, $25 \%$ when they believe that none of the two others will pay, $33.33 \%$ when they believe that one will pay but $100 \%$ when they believe that the two others will pay.
} 
Model (2) shows that a least performer is $24.13 \%$ more likely to choose to pay the fee than a subject who receives the second rank, with no difference between subjects who get ranks 1 and 2, respectively. This again supports Conjecture 1. Second, models (1) and (2) indicate that being in a triad with in-groups only (i.e., in the Homogeneous treatment) or with out-groups (i.e., in the Heterogeneous treatment) does not affect the willingness to pay the fee as compared to the Minimal Identity treatment, controlling or not for the beliefs about others' willingness to pay the fee. In contrast, in the No Identity treatment, the willingness to pay the fee is reduced by $16.19 \%$ compared to the Minimal Identity treatment. The difference between the No Identity and the Minimal Identity treatments is, however, not significant for the least performers (model (3)).

The third finding relates to the triad configuration in terms of group identity in the Heterogeneous treatment. In this treatment, neither the least performers (model (3)) nor the other members (models (4) and (5)) condition their decision to pay on the triad composition. This does not support Conjecture 3.

The fourth finding is related to beliefs. While model (1) shows that eliciting beliefs did not change subjects' behavior, all the other models find that subjects' willingness to help increases in the number of triad members they believe will also pay. For example, model (2) shows that, compared to the condition where we did not elicit beliefs, predicting that no one will pay decreases one's willingness to pay by $29.30 \%$, predicting that one other member will pay increases it by $19.76 \%$, and predicting that the two other members will pay even increases it by $62.95 \%$. For the least performers (model (3)), these percentages are $-26.89 \%, 40.41 \%$ and $46.23 \%$, respectively. For the best and medium performers (model (4)), believing that one other member will pay has no impact, but believing that the two others will pay increases one's own willingness to pay by $57.76 \%$, while believing that no one is paying reduces this willingness by $22.09 \%$. This rejects an interpretation in terms of coordination which would require that one is more willing to pay when expecting that a single other member will sacrifice. Our finding suggests instead that subjects who think that several others are willing to pay are also more willing to sacrifice because image has an important value and so, preserving it in a group is a 
norm. $^{20}$

We summarize these findings as follows:

Result 1: A large majority of individuals sacrifice resources to avoid public exposure and their motivation is stronger when the beliefs about the intensity of help expected from others are higher.

Result 2: A smaller majority of individuals are willing to sacrifice to waive the exposure of other people.

Results 1 and 2 support Conjecture 1.

Result 3: Even when there is no group identity, more than one third of subjects are still willing to preserve others' face. But this is lower than in the treatments with group identity. In contrast, increasing the saliency of group identity by means of labels has little impact on behavior.

This result does not support Conjecture 3.

\subsection{Emotions}

In this section we explore whether the strong willingness to preserve one's and others' face is driven by the fact that the ranking of subjects and the threat of exposure generate more unpleasant and less pleasurable emotions. Figures 1 and 2 display the evolution of self-reported levels of shame and happiness over time, by subjects' rank in part 3, and according to actual public exposure of the least performers. We consider the feelings reported at the beginning of the session, after receiving feedback on one's score in part 1, after receiving feedback on scores and ranks in part 2, after deciding on paying the fee in part 3, after the public exposure of the least performers, and after the feedback on scores and ranks in part 4. All non-parametric tests reported here are two-sided and consider each individual as an independent observation.

\section{(Insert Figures 1 and 2 about here)}

The figures show no difference in shame and happiness between the categories of subjects at the beginning of the session or when receiving feedback in part 1 (Mann-Whitney tests - MW

\footnotetext{
${ }^{20}$ In other regressions not reported here but available upon request, we also controlled for the difference in scores between the player and the least performer in the triad. The coefficient of this variable is never significant. Helping to save face is not conditional on differences in effort.
} 
hereafter,$- p>0.010$ in pairwise comparisons). When learning their rank in part 2 , the least performers in part 3 start experiencing more shame and less happiness than subjects with rank 1 or $2(\mathrm{MW}, p<0.001)$, while the best performers in part 3 start experiencing more happiness than the others $(\mathrm{MW}, p<0.001){ }^{21}$ The figures show that the least performers' emotions are exacerbated in part 3 when learning their risk of being exposed. In this part too, the differences with the levels of emotions reported by the other players are significant ( $\mathrm{MW}, p<0.001)$.

The figures reveal three interesting findings about the emotions experienced after public exposure. First, the publicly exposed subjects report a level of shame similar to the level reported before knowing the decision of their triad members (Wilcoxon test $-\mathrm{W}$ hereafter,$- p=0.522$ ); this suggests that anticipatory feelings are as strong as those experienced at the time of exposure. Their level of shame decreases in part 4 compared to the moment of exposure (W test, $p=0.008$ ) without returning to its initial level when receiving the same type of feedback, such as in part 2 (W test, $p=0.033$ ), suggesting that it takes time to dissipate. Second, the least performers who have not been exposed experience an immediate decrease of their shame intensity at the time of exposure compared to when waiting for the decision of their triad members (W test, $p<0.001$ ) while their level of happiness peaks and exceeds that reported by the best performers (MW test, $p=0.005)$. In part 4 they still report a level of happiness that is higher than that reported by those who have been exposed (MW test, $p=0.004$ ). They may feel satisfaction from seeing that others helped them. Finally, the best performers in part 3 report a sharp decrease in happiness when the least performers are publicly exposed compared to when they decide to pay the fee (W test, $p<0.001)$, which suggests empathy.

Of course, these feelings could be driven by the fact that individuals dislike being singled out, independently of any signaling value of a lower relative performance. Two elements suggest that this interpretation is not the most likely. First, the least performers' shame increases and their happiness decreases already between part 1 (when receiving feedback on own score) and part 2

\footnotetext{
21 There is indeed a relative stability in ranks across parts. $63 \%$ of the best performers in part 3 received already rank 1 in part $1,74 \%$ received rank 1 both in part 2 and in part $4.65 \%$ of the least performers in part 3 received already rank 3 in part $1,76 \%$ in part 2 , and $74 \%$ in part 4.
} 
(when learning also the rank) (W tests, both $p<0.001$ ), signaling that a lower rank generates a change in emotions. Second, we ran two sessions of a game similar to the Minimal Identity treatment, except that it is the best performer who is publicly exposed unless at least one player pays to avoid exposure $(\mathrm{N}=36)$. In this treatment the percentage of best performers paying to avoid public praise is $33.33 \%$ (4 out of 12). A binomial test indicates that the probability to pay the fee for the least performers in the Minimal Identity treatment is significantly higher than this percentage $(p<0.001)$. We can reasonably conclude that what people try to avoid is mainly the exposure of a lower performance.

We summarize our analysis as follows:

Result 4: Both the individuals threatened by potential exposure and those actually exposed express more shame and decreased happiness. Subjects who are not threatened also report a decrease in happiness at the moment of exposure. Empathy may be the mechanism behind the decision to sacrifice to preserve others' face.

\subsection{Evolution of performance levels}

To study whether image influences performance, and thus efficiency, Table 4 displays descriptive statistics on the mean individual performance in each part in the No Feedback treatment (with no social information and no exposure) and in each other treatment for all subjects, and then only for the least performers in part 3 according to whether they were or not exposed (excluding the No Feedback treatment). As a complement, Table A1 in Appendix 3 reports the mean total performance at the triad level in each part and each treatment depending on whether the least performer in the triad was or not exposed in part 3; it should in particular reveal whether exposure has or not a negative effect on the evolution of the total performance of triads between parts 3 and 4.

\section{(Insert Table 4 about here)}

Table 4 attests of the presence of intrinsic motivation since subjects provide a positive level of effort in all parts although they are paid a fixed wage. Individual performance increases from 
part 1 to part 2 and from part 2 to part 3 in each treatment (W tests, $p<0.001$ ). This cannot be the result of the pressure of social comparisons and the risk of exposure since the same evolution is observed in the No Feedback treatment. We find no significant difference in performance between the No Feedback and each other treatment in any part (MW tests, $p>0.100$ ). Moreover, the mean individual performance is not higher in the Heterogeneous than in the Homogenous treatment in any part (MW tests, $p>0.100)$. Being matched with out-groups does not boost effort in part 3 to increase the negative externalities of one's effort on out-groups. This analysis does not support Conjecture 4.

The evolution of individual performance between parts 3 and 4 is more diverse. Although there is no longer any risk of exposure, individual mean performance is higher in part 4 than in part 3 in the Minimal Identity treatment and the Heterogeneous treatments (W tests, $p<0.001$ ), but not in the other treatments $(p>0.100)$. Interestingly, when we pool all the treatments (excluding the No Feedback treatment), we find that both the subjects with ranks 1 or 2 and the least performers who have not been exposed in part 3 increase their effort in part $4(p<0.001)$, but not the least performers who have been exposed $(p=0.626)$. This observation suggests that actual exposure tends to crowd-out intrinsic motivation or that public exposure left people uncomfortable and distracted, which reduced their performance in the last part. Table A1 in Appendix 3 also indicates that in both the Heterogeneous and the No Identity treatments, the mean performance of the triads increases between parts 3 and 4 when the least performer has not been exposed while it decreases when the least performer has been exposed. Wilcoxon tests comparing mean performance at the triad level in parts 3 and 4 indicate, however, that these effects are not significant ( $p>0.18$ in all treatments, see Appendix 3).

Table 5 reports the results of OLS regressions. In model (1), the dependent variable is the difference in the sum of performance levels at the triad level between part 4 and part 3. In model (2) it is the difference in the individual performance between part 4 and part 3 for the subjects who got rank 1 or 2. Model (3) is for the least performers. In all models, the independent variables include dummy variables for each treatment, with the No Feedback treatment as the 
reference category, and an indicator of whether the least performer in the triad has been exposed. ${ }^{22}$ In models (2) and (3), they also include a dummy variable indicating whether or not the subject chose to pay the fee and the same demographic variables as in Table 3 . Standard errors are clustered at the triad level in model (2).

In contrast with the non-parametric statistics reported below Table A1, model (1) indicates that the evolution of the total performance of triads is significantly and negatively affected by the exposure of the least performer in part 3. Model (2) identifies no effect of the exposure of the least performer on the evolution of performance of the subjects who got rank 1 or 2 . In contrast, model (3) reveals a significant negative effect of exposure in part 3 on the performance of the least performers in part 4, suggesting a decrease in their intrinsic motivation. Indeed, the performance of the exposed subjects decreases by 3.12 units between parts 3 and 4 compared to those who have been able to avoid exposure; this drives the negative tendency observed at the triad level in model (1). This partially supports Conjecture 2 and leads to our final result.

\section{(Insert Table 5 about here)}

Result 5: While social information on relative performance and group identity in general have little impact on performance, public exposure crowds-out the future intrinsic motivation of the least performers who have been exposed.

This result partially supports Conjecture 2 and rejects Conjecture 4.

\section{DISCUSSION AND CONCLUSION}

The importance of preserving self and others' image in social interactions is well known from negotiations and diplomacy, but it has received little attention in economics. Our experiment shows that most people are willing to forego some money to avoid the public display of a poor relative performance. We interpret this behavior as evidence of the fear of losing face since

\footnotetext{
22 These two dummy variables take value 0 in the No Feedback treatment. We do not include the intensity of shame and happiness feelings in part 3 because they are not random variables and would create an endogeneity issue. We also tested models (2) and (3) after including a dummy variable indicating whether the exposed individual belongs to the minority in the Heterogeneous treatment. This variable was not significant. Therefore, we do not report these additional regressions.
} 
individuals report shame and a lower level of happiness when they learn about their lower rank. We can exclude that this behavior captures only distaste for exposure, because when it is the best performer who is exposed, a much lower proportion of players are willing to avoid it. This result is striking since subjects were paid a fixed wage, the task we used is trivial, and performance helps no one. If people care about being exposed, this indicates that when there is intrinsic motivation the public exposure of a low rank is associated with a devaluated perception of the self in society. These findings suggest that anticipatory negative emotions inflict a moral cost to individuals that is higher than the monetary cost incurred to avoid exposure.

A second major finding is that a majority of people sacrifice resources to preserve others' face although there is no indication of future personal benefits from this action. This empathetic behavior suggests that altruism extends beyond the monetary realm, as individuals like to do good to others also in terms of image. In our experiment the cost incurred to avoid others' exposure represents $10 \%$ of the income. It would be interesting to vary this cost in order to elicit the value of others' face. In an extension of the paper we could also measure precisely the willingness to pay for saving face, using the Becker-DeGroot-Marschak mechanism. Another possible extension would be to allow people to pay to avoid the exposure of the least performer in another triad in order to identify to what extent feeling responsible for the rank of the least performer contributes to the willingness to pay to save others' face.

Even in the No Identity treatment where triads are short-lived, more than one third of the subjects sacrifice resources for preserving others' image. But the difference between the proportions in this treatment and in the three other treatments with more or less salient group identity indicates that saving face is not a simple moral norm. The main difference in the decision to sacrifice is not between the treatments in which painting identity is salient and the Minimal Identity treatment, but between the three treatments with fixed triads and the No Identity treatment. This difference may result from the combination of a higher social distance between subjects in the No Identity treatment and a lower concern for reputation for future coordination since triads are short-lived. Of course, subjects are not aware of the content of the next part in the 
experiment when they decide whether or not to pay the fee, but they know that they will remain matched with the same persons until the end of the experiment. Teasing out the impact of social distance and reputation concerns suggests possible extensions. Rematching triads in the Homogeneous/Heterogeneous treatments in each part or allowing subjects to pay to avoid the exposure of an in-group or an out-group least performer in another triad would get rid of reputation concerns ${ }^{23}$.

Our manipulation of the saliency of group identity in fixed triads has no direct effect on behavior. This result is in contrast to previous studies that have found an impact of the saliency of group identity (Eckel and Grossman, 2005; Charness et al., 2007; Chen and Chen, 2011). This may be due to the fact that in-group favoritism when triads are fixed would be perceived as a violation of a norm (Harris et al., 2015). It may also result from the fact that we conducted the study in China. Buchan et al. (2009) found that Chinese subjects sent and returned significantly more to out-groups than to in-groups in a trust game, while the opposite was found for American subjects. It may be more difficult to create a group identity in a collectivist culture where people have fewer in-groups than in individualistic societies (Triandis, 1995). In collectivist cultures (Hofstede, 1980), the goals of the group matter more than those of individuals, which could lead collectively oriented subjects to pay less attention to social distance. Another interpretation is that using the Klee-Kandinsky paintings as a support for generating a salient group identity may not be as effective in China as in occidental countries because individuals are less familiar with these painters. These interpretations are not, however, consistent with the fact that the subjects in our experiment report a relatively strong feeling of group membership. Although a few subjects mentioned during the group identity formation stage that they were not familiar with the style of the paintings, almost all subjects discussed with their group members about the differences between paintings regarding colors and shapes. Overall, a majority of subjects made two correct guesses about the two paintings in the group-solving task $(69.05 \%$ in the Homogeneous treatment and $73.02 \%$ in the Heterogeneous treatment, which is significantly different from a random

${ }^{23}$ We thank an anonymous reviewer for these suggestions. 
response; binomial tests, $p<0.001$ ). This suggests that the chat was useful and contributed to the building of group identity. Cross-cultural replication studies would allow us to test whether in individualistic cultures people are less willing to sacrifice to save others' face and whether social distance has a stronger impact on this decision. Our expectation is that people would still sacrifice to help others, as previous studies in social sciences have shown that face maintenance is important also in Western cultures (Ting-Toomey et al., 1991).

Finally, we found some evidence that public exposure tends to crowd-out future intrinsic motivation of individuals who had been publicly exposed as poor performers, at least in the short run. This suggests that providing feedback about relative performance may sometimes encourage people to work harder, but when information is disseminated it also generates negative emotions, the longer-term effects of which on individuals' performance are still to be explored. We acknowledge that in our experiment, ranking occurs periodically whereas shaming occurs once. In real settings, public exposure of relative performance may last longer and thus, our results on the importance of face and its impact on the motivation and the performance of the least performers may be underestimated by design. It would make sense to study whether individuals who feel they have lost face because of public rankings become less willing to identify themselves with their group in future interactions. In this experiment, we only considered the case when shaming affects an individual, but shaming may be collective when it involves a ranking of organizations (schools, universities, companies). It would be interesting to explore how shaming affects efficiency and through which mechanisms when it is collective rather than individual, as in this case the loss of utility may derive from a lower identification to the group than from a downgraded image. This is left for further investigation.

\section{REFERENCES}

Abbink, Klaus, and Dona Harris, 2012. In-group Favouritism and Out-group Discrimination in Naturally Occurring Groups, Discussion Paper 616, University of Oxford.

Akerlof, George A. and Rachel E. Kranton, 2000. Economics and Identity, Quarterly Journal of 
Economics, 115(3), 715-53.

Andreoni, James, and John Miller, 2002. Giving According to GARP: An Experimental Test of the Consistency of Preferences for Altruism. Econometrica, 70(2), 737-753.

Alpizar, Francisco, Fredrik Carlsson, and Olof Johansson-Stenman, 2008. Anonymity, Reciprocity and Conformity: Evidence from Voluntary Contributions to a National Park in Costa Rica, Journal of Public Economics, 92(5-6), 1047-1060.

Ariely, Dan, Anat Bracha, and Stephan Meier. 2009. Doing Good or Doing Well? Image Motivation and Monetary Incentives in Behaving Prosocially, American Economic Review, 99(1), 544-555.

Barankay, Iwan, 2012. Rank Incentives: Evidence from a Randomized Workplace Experiment, Working Paper, University of Pennsylvania.

Baumeister, Roy F., Jennifer D. Campbell, Joachim I. Krueger and Kathleen D. Vohs, 2005. Exploding the Self-Esteem Myth, Scientific American, 292, 84-91.

Benabou, Roland, and Jean Tirole, 2006. Incentives and Prosocial Behavior, American Economic Review, 96(5), 1652-1678.

Ben-Ner, Avner, Brian P. McCall, Stephane Massoud, and Hua Wang, 2009. Identity and In-group/Out-group Differentiation in Work and Giving Behaviors: Experimental evidence, Journal of Economic Behavior \& Organization, 72, 153-170.

Beresford, Alastair, Dorothea Kübler, and Sören Preibusch, 2012. Unwillingness to Pay for Privacy: A Field Experiment, Economics Letters, 117(1), 25-27.

Bernhard, Helen, Ernst Fehr, and Urs Fischbacher, 2006. Group Affiliation and Altruistic Norm Enforcement, American Economic Review, 96(2), 217-221.

Bohnet, Iris, and Bruno Frey, 1999. The Sound of Silence in Prisoner's Dilemma and Dictator Games, Journal of Economic Behavior \& Organization, 38 (1), 43-57.

Bowles, Samuel, and Herbert Gintis, 2004. Persistent Parochialism: Trust and Exclusion in Ethnic Networks, Journal of Economic Behavior \& Organization, 55(1), 1-23.

Bradler, Christiane, Robert Dur, Susanne Neckermann, and Arjan Non, 2013. Employee Recognition and Performance: A Field Experiment, CESifo Working Paper 4164. Available at SSRN: http://ssrn.com/abstract=2243458

Buchan, Nancy R., Rachel Croson, and Eric J. Johnson. 2006. Let's Get Personal: An International Examination of the Influence of Communication, Culture and Social Distance on Other Regarding Preferences, Journal of Economic Behavior \& Organization, 60, 373-398.

Cappelen, Alexander W., Trond Halvorsen, Erik Ø. Sørensen, and Bertil Tugodden, 2012. Face-saving or Fair-minded: What Motivates Moral Behavior?, Working Paper, Norwegian School of Economics, Bergen. 
Chakravarty, Surajeet, and Miguel A. Fonseca, 2011. The Effect of Social Fragmentation on Public Good Provision: An Experimental Study, Discussion Paper 1207, Exeter University.

Charness, Gary, Luca Rigotti, and Aldo Rustichini, 2007. Individual Behavior and Group Membership, American Economic Review, 97(4), 1340-1352.

Charness, Gary, and Uri Gneezy, 2008. What's in a Name? Anonymity and Social Distance in Dictator and Ultimatum Games, Journal of Economic Behavior \& Organization, 68 (1), 29-35.

Charness, Gary, David Masclet, and Marie Claire Villeval, 2014. The Dark Side of Competition for Status, Management Science, 60(1), 38-55.

Chen, Roy, and Yan Chen, 2011. The Potential of Social Identity for Equilibrium Selection, American Economic Review, 101(6), 2562-2589.

Chen, Yan, and Sherry Xin Li, 2006. Group Identity and Social Preferences, American Economic Review, 99(1), 431-457.

Coricelli, Giorgio, Mateus Joffily, Claude Montmarquette, and Marie Claire Villeval, 2010. Cheating, Emotions, and Rationality: An Experiment on Tax Evasion, Experimental Economics, 13, 226-247.

Coricelli, Giorgio, Elena Rusconi, and Marie Claire Villeval, 2014. Tax Evasion and Emotions in Repeated Interactions: An Empirical Test of Re-integrative Shaming Theory, Journal of Economic Psychology, 40, 49-61.

Eckel, Catherine C., and Philip Grossman, 2005. Managing Diversity by Creating Team Identity, Journal of Economic Behavior and Organization, 58(3), 371-392.

Eckel, Catherine C., Enrique Fatas, and Rick Wilson, 2010. Cooperation and Status in Organizations, Journal of Public Economic Theory, 12(4), 737-762.

Ellingsen, Tore, and Magnus Johannesson, 2007. Paying Respect, Journal of Economic Perspectives, 21(4), 135-149.

Ellingsen, Tore, and Magnus Johannesson, 2008a. Pride and Prejudice: The Human Side of Incentive Theory, American Economic Review, 98(3), 990-1008.

Ellingsen, Tore, and Magnus Johannesson, 2008b. Anticipated Verbal Feedback Induces Altruistic Behaviour, Evolution and Human Behaviour, 29(2), 100-105.

Eriksson, Tor, and Marie Claire Villeval, 2011. Respect and Relational Contracts, Journal of Economic Behavior \& Organization, 81, 286-298.

Ewers, Mara, and Florian Zimmermann, 2015. Image and Misreporting, Journal of the European Economic Association 13(2), 363-380.

Falk, Armin, and Urs Fischbacher, 2006. A Theory of Reciprocity, Games and Economic Behavior, 54(2), 293-315. 
Fehr, Ernst, and Urs Fischbacher, 2003. The Nature of Human Altruism, Nature, 425, 785-791.

Feri, Francesco, Caterina Gianetti, and Nicola Jentzsch, 2013. Disclosure of Personal Information Under Risk of Privacy Shocks, mimeo, DIW Berlin.

Fershtman, Chaim, and Uri Gneezy, 2001. Discrimination in a Segmented Society: An Experimental Approach, Quarterly Journal of Economics, 116(1), 351-377.

Galeotti, Fabio, and Daniel J. Zizzo (2014). What Happens if You Single out? An Experiment, Social Choice and Welfare, 43(3), 703-729.

Goette, Lorenz, David Huffman and Stephan Meier, 2006. The Impact of Group Membership on Cooperation and Norm Enforcement: Evidence Using Random Assignment to Real Social Groups, American Economic Review, 96(2), 212-216.

Goette, Lorenz, David Huffman and Stephan Meier, 2012a. The Impact of Social Ties on Group Interactions: Evidence from Minimal Groups and Randomly Assigned Real Groups, American Economic Journal: Microeconomics, 4(1), 101-115.

Goette, Lorenz, David Huffman, Stephan Meier, and Matthias Sutter, 2012b. Competition Between Organizational Groups: Its Impact on Altruistic and Anti-Social Motivations, Management Science, 58(5), 948-960.

Goffman, Erving (2005). Interaction Ritual: Essays in Face-to-Face Behavior. Aldine Transaction.

Guala, Francesco, Luigi Mittone, and Matteo Ploner, 2013. Group Membership, Team Preferences and Expectations, Journal of Economic Behavior \& Organization, 86, 183-190.

Hargreaves-Heap, Shaun P., and Daniel J. Zizzo, 2009. The Value of Groups, American Economic Review, 99(1), 295-323.

Harris, Donna, Benedikt Herrmann, and Andreas Kontoleon, 2009. Two's Company, There's a Group: The Impact of Group Identity and Group Size on In-Group Favouritism, CeDex Discussion Paper 2009-13, Nottingham.

Harris, D., Herrmann, B., Kontoleon, A., \& Newton, J. (2015). Is it a norm to favour your own group? Experimental Economics 18(3), 491-521.

Herbst, Luisa, Kai A. Konrad, and Florian Morath, 2012. Endogenous Group Formation in Experimental Contests, Working Paper of the Max Planck Institute for Tax Law and Public Finance 2012-10. Available at SSRN: http://ssrn.com/abstract=2166206.

Ho, David Yau-Fai, 1976. On the Concept of Face, American Journal of Sociology, 81(4), 867-884.

Hofstede, Geert H., 1980. Culture’s Consequences. Berkeley: University of California Press.

Hopfensitz, Astrid, and Ernesto Reuben, 2009. The Importance of Emotions for the Effectiveness 
of Social Punishment, Economic Journal, 119(540), 1534-59.

$\mathrm{Hu}$, Hsien Chin, 1944. The Chinese Concept of 'Face', American Anthropologist, 46 (1), 45-64.

Hugh-Jones, David, and David Reinstein, 2010. Losing Face, Jena Working Paper 2013-068.

Johansson-Stenman, Olof, and Henrik Svedsater, 2012. Self-image and Valuation of Moral Goods: Stated versus Real Willingness to Pay, Journal of Economic Behavior \& Organization, 84(3), 879-891.

Johansson-Stenman, Olof, and Peter Martinsson, 2006. Honestly, Why are you Driving a BMW? Journal of Economic Behavior \& Organization, 60(2), 129-146.

Kato, Takao, and Pian Shu, 2013. Competition and Social Identity in the Workplace: Evidence from a Chinese Textile Firm, Harvard Business School Working Paper 14-011.

Kollock, Peter, 1998. Transforming Social Dilemmas: Group Identity and Cooperation, in Peter A. Danielson, ed., Modeling Rationality, Morality and Evolution. Oxford: Oxford University Press, 186-210.

Kosfeld, Michael, and Susanne Neckermann, 2011. Getting more Work for Nothing? Symbolic Awards and Worker Performance, American Economic Journal: Microeconomics, 3(1), 1-16.

Koszegi, Botond, 2006. Ego Utility, Overconfidence and Task Choice, Journal of the European Economic Association, 4(4), 673-707.

Kuhnen, Camelia M., and Agnieszka Tymula, 2012. Feedback, Self-Esteem and Performance in Organizations, Management Science, 58, 94-113.

Lacetera, Nicola, and Mario Macis, 2010. Social Image Concerns and Prosocial Behavior: Field Evidence from a Nonlinear Incentive Scheme, Journal of Economic Behavior \& Organization, 76(2), 225-237.

Masella, Paolo, Stephen Meier, and Philipp Zahn, 2014. Incentives and Group Identity, Games and Economic Behavior, 86, 12-25.

Mruk, Christopher J., 2006. Self-Esteem Research, Theory, and Practice: Toward a Positive Psychology of Self-Esteem. 3rd Edition. New-York: Springer.

Qi, Xiaoying (2011). Face: A Chinese Concept in a Global Sociology, Journal of Sociology, 47, 279-296.

Redding, S. Gordon, and Michael Ng, 1982. The Role of 'Face' in the Organizational Perceptions of Chinese Managers, Organization Studies, 3(3), 201-219.

Smith, Richard H., J. Matthew Webster, W. Gerrod Parrott, and Heidi L. Eyre, 2002. The Role of Public Exposure in Moral and Non moral Shame and Guilt, Journal of Personality and Social Psychology, 83(1), 138-159.

Spencer, Steven J., Steven Fein, Christine D. Lomore, 2001. Maintaining One's Self-Image Vis-à-Vis Others: The Role of Self-Affirmation in the Social Evaluation of the Self, 
Motivation and Emotion, 25(1), 41-65.

Spencer-Oatey, Helen, 2007. Theories of Identity and the Analysis of Face, Journal of Pragmatics, 39(4), 639-656.

Tajfel, Henri, Michael G. Billig, Robert P. Bundy, and Claude Flament, 1971. Social Categorization and Intergroup Behavior, European Journal of Social Psychology, 1(2), 149-178.

Tajfel, Henri, and John Turner, 1979. An Integrative Theory of Intergroup Conflict, in William Austin and Stephen Worchel, Eds., The Social Psychology of Intergroup Relations, Monterey: CA, Brooks/Cole.

Ting-Toomey, Stella, 2005. The Matrix of Face: An Updated Face-Negotiation Theory, in W.B. Gudykunst, ed., Theorizing About Intercultural Communication. Thousand Oaks: Sage, 71-92.

Ting-Toomey, Stella, Ge Gao, Paula Trubiski, Zhizhong Yang, Hak Soo Kim, Sung-Ling Lin, Tsukasa Nishida. 1991. Culture, Face Maintenance, and Styles of Handling Interpersonal Conflict: A Study in Five Cultures, International Journal of Conflict Management, 2(4), 275 296.

Tran, Ahn, and Richard Zeckhauser, 2012. Rank as an Incentive: Evidence from a Field Experiment, Mimeo Harvard University.

Triandis, H.C. (1995). Individualism and Collectivism, Westview Press.

Xiao, Erte, and Daniel Houser, 2011. Punish in Public, Journal of Public Economics, 95(7-8), 1006-1017.

Yang, Kuo-Shu (ed.), 1989. The Psychology of the Chinese. Taipei: Kui-Kuan Books.

Zizzo, Daniel J., 2011. You are not in my Boat: Common Fate and Discrimination against Outgroup Members, International Review of Economics, 58, 91-103. 


\section{TABLES AND FIGURES}

Table 1. Summary of sessions

\begin{tabular}{lccccc}
\hline \multicolumn{1}{c}{ Treatments } & \multicolumn{2}{c}{$\begin{array}{c}\text { Number of sessions } \\
\text { With belief elicitation }\end{array}$} & $\begin{array}{c}\text { Number of } \\
\text { participants }\end{array}$ & $\begin{array}{c}\text { Mean age } \\
\text { (years) }\end{array}$ & \% males \\
\hline Minimal Identity & 4 & 2 & 72 & 22.24 & 15.28 \\
Homogenous & 7 & 4 & 126 & 21.91 & 26.98 \\
Heterogeneous & 7 & 4 & 126 & 22.25 & 28.57 \\
No Identity & 4 & 4 & 72 & 22.19 & 29.17 \\
No Feedback & 2 & 0 & 24 & 21.25 & 20.83 \\
\hline Total & 24 & 14 & 420 & 22.09 & $25.48 \%$ \\
\hline \hline
\end{tabular}


Table 2. Percentages of participants paying the fee to avoid the public exposure of the least performers, by treatment and by performance rank, and actual exposure

\begin{tabular}{lcccc}
\hline Treatments & $\begin{array}{c}\text { Rank 1 } \\
\text { in part 3 }\end{array}$ & $\begin{array}{c}\text { Rank 2 } \\
\text { in part 3 }\end{array}$ & $\begin{array}{c}\text { Rank 3 } \\
\text { in part 3 }\end{array}$ & $\begin{array}{c}\text { \% of least } \\
\text { performers } \\
\text { exposed }\end{array}$ \\
\hline Minimal Identity & $54.17 \%(13 / 24)$ & $54.17 \%(13 / 24)$ & $75.00 \%(18 / 24)$ & $37.50 \%(9 / 24)$ \\
Homogenous & $50.00 \%(21 / 42)$ & $66.67 \%(28 / 42)$ & $80.95 \%(34 / 42)$ & $28.57 \%(12 / 42)$ \\
Heterogeneous & $57.14 \%(24 / 42)$ & $54.76 \%(23 / 42)$ & $71.43 \%(30 / 42)$ & $33.33 \%(14 / 42)$ \\
$\quad$ - KKk or kkK & $33.33 \%(3 / 9)$ & $44.44 \%(4 / 9)$ & $66.67 \%(6 / 9)$ & $44.44 \%(4 / 9)$ \\
$\quad$ - Kkk or kKK & $69.23 \%(9 / 13)$ & $61.54 \%(8 / 13)$ & $69.23 \%(9 / 13)$ & $30.00 \%(6 / 13)$ \\
$\quad$ - KkK or kKk & $60 \%(12 / 20)$ & $55 \%(11 / 20)$ & $75 \%(15 / 20)$ & $20 \%(4 / 20)$ \\
No Identity & $33.33 \%(8 / 24)$ & $41.67 \%(10 / 24)$ & $62.50 \%(15 / 24)$ & $66.67 \%(16 / 24)$ \\
\hline Total & $50.00 \%(66 / 132)$ & $56.06 \%(74 / 132)$ & $73.48 \%(97 / 132)$ & $38.64 \%(51 / 132)$ \\
\hline
\end{tabular}

Note: "K" is for the Kandinsky group, "k" for Klee group. "KKk or kkK" reads as follows: in the triad, the participants who get the first and the second ranks have the same group identity while the least performer has another group identity. 
Table 3. Determinants of the decision to pay the fee to avoid the public exposure of the least performer

\begin{tabular}{|c|c|c|c|c|c|}
\hline \multirow{2}{*}{$\begin{array}{l}\text { Dependent variable: } \\
\text { Decision to pay the fee }\end{array}$} & \multicolumn{5}{|c|}{ Probit models } \\
\hline & $\begin{array}{c}\text { All } \\
\text { subjects } \\
(1)\end{array}$ & $\begin{array}{c}\text { All } \\
\text { subjects } \\
(2)\end{array}$ & $\begin{array}{c}\text { Rank } 3 \\
\text { only } \\
(3)\end{array}$ & $\begin{array}{c}\text { Ranks } \\
1 \text { and } 2 \\
(4)\end{array}$ & $\begin{array}{c}\text { Ranks } 1 \text { and } \\
2 \text { - Hetero. T. } \\
\text { (5) }\end{array}$ \\
\hline Minimal Identity treatment & Ref. & Ref. & Ref. & Ref. & - \\
\hline Homogenous treatment & $\begin{array}{c}0.068 \\
(0.070)\end{array}$ & $\begin{array}{c}0.033 \\
(0.058)\end{array}$ & $\begin{array}{c}0.077 \\
(0.101)\end{array}$ & $\begin{array}{c}0.022 \\
(0.075)\end{array}$ & - \\
\hline Heterogeneous treatment & $\begin{array}{l}-0.001 \\
(0.066)\end{array}$ & $\begin{array}{c}0.036 \\
(0.058)\end{array}$ & $\begin{array}{c}0.004 \\
(0.105)\end{array}$ & $\begin{array}{c}0.069 \\
(0.090)\end{array}$ & - \\
\hline No Identity treatment & $\begin{array}{c}-0.166^{* *} \\
(0.083)\end{array}$ & $\begin{array}{l}-0.162 * * \\
(0.075)\end{array}$ & $\begin{array}{l}-0.048 \\
(0.120)\end{array}$ & $\begin{array}{c}-0.225^{* *} \\
(0.104)\end{array}$ & - \\
\hline$i$ is Rank 2 in part 3 & Ref. & Ref. & - & Ref. & Ref. \\
\hline$i$ is Rank 1 in part 3 & $\begin{array}{l}-0.060 \\
(0.051)\end{array}$ & $\begin{array}{l}-0.071 \\
(0.046)\end{array}$ & - & $\begin{array}{l}-0.071 \\
(0.049)\end{array}$ & $\begin{array}{c}0.055 \\
(0.101)\end{array}$ \\
\hline$i$ is Rank 3 in part 3 & $\begin{array}{c}0.162 * * * \\
(0.056)\end{array}$ & $\begin{array}{c}0.241 * * * \\
(0.052)\end{array}$ & - & - & - \\
\hline $\begin{array}{l}\text { Heterogeneous T. } * \text { Rank } 3 \\
\text { is an out-group }\end{array}$ & - & $\begin{array}{l}- \\
-\end{array}$ & - & $\begin{array}{c}0.001 \\
(0.098)\end{array}$ & - \\
\hline Rank 3 is is in-group & - & - & - & - & Ref. \\
\hline Rank 3 is the only out-group & - & - & - & - & $\begin{array}{l}-0.091 \\
(0.131)\end{array}$ \\
\hline $\begin{array}{l}\text { Heterogeneous } \mathrm{T} . * \\
i \text { matched with } 2 \text { out-groups }\end{array}$ & - & - & $\begin{array}{c}0.007 \\
(0.135)\end{array}$ & - & $\begin{array}{l}0.090 \\
(0.111)\end{array}$ \\
\hline Belief elicitation & $\begin{array}{c}0.042 \\
(0.056)\end{array}$ & $\begin{array}{c}-0.293 * * * \\
(0.075)\end{array}$ & $\begin{array}{c}-0.269 * * * \\
(0.085)\end{array}$ & $\begin{array}{l}-0.221 * * \\
(0.108)\end{array}$ & $\begin{array}{c}-0.504 * * * \\
(0.168)\end{array}$ \\
\hline $\begin{array}{l}\text { Belief elicitation } * \\
\text { Belief: } 1 \text { other pays }\end{array}$ & - & $\begin{array}{c}0.198 * * * \\
(0.071)\end{array}$ & $\begin{array}{c}0.404 * * * \\
(0.083)\end{array}$ & $\begin{array}{l}-0.010 \\
(0.122)\end{array}$ & - \\
\hline $\begin{array}{l}\text { Belief elicitation } * \\
\text { Belief: } 2 \text { others pay }\end{array}$ & - & $\begin{array}{c}0.630 * * * \\
(0.067)\end{array}$ & $\begin{array}{c}0.462 * * * \\
(0.106)\end{array}$ & $\begin{array}{c}0.578 * * * \\
(0.100)\end{array}$ & - \\
\hline $\begin{array}{l}\text { Belief elicitation * } \\
\text { Belief nb others pay }\end{array}$ & - & - & - & - & $\begin{array}{c}0.463 * * * \\
(0.091)\end{array}$ \\
\hline Demographics & Yes & Yes & Yes & Yes & Yes \\
\hline $\mathrm{N}$ & 394 & 394 & 132 & 262 & 84 \\
\hline Log-likelihood & -249.638 & -210.279 & -61.440 & -138.295 & -45.081 \\
\hline $\mathrm{LR} \mathrm{Chi}^{2}$ & 29.70 & 91.19 & 29.81 & 82.00 & 23.50 \\
\hline Prob $>\mathrm{Chi}^{2}$ & $<0.001$ & $<0.001$ & $<0.001$ & $<0.001$ & 0.002 \\
\hline Pseudo $\mathrm{R}^{2}$ & 0.058 & 0.206 & 0.195 & 0.236 & 0.218 \\
\hline
\end{tabular}

Notes: The Table reports marginal effects. Standard errors are clustered at the triad level, except in model (3). 
In models (1), (2) and (4), two observations are missing because the level of wealth has not been recorded. *** indicate significance at the $1 \%$ level, $* *$ at the $5 \%$ level and * at the $10 \%$ level. In models (1), (2) and (4), two observations are missing because the level of wealth has not been recorded.

Table 4. Mean performance, by part and by treatment

\begin{tabular}{lccccc}
\hline & Part 1 & Part 2 & Part 3 & Part 4 & N \\
\hline No Feedback treatment & $128.54(16.09)$ & $133.50(17.16)$ & $138.58(15.34)$ & $140.17(13.01)$ & 24 \\
\hline Minimal Identity treatment & $127.14(11.41)$ & $132.56(14.93)$ & $136.08(16.64)$ & $138.86(17.64)$ & 72 \\
Homogenous treatment & $126.61(15.36)$ & $135.28(14.82)$ & $140.10(13.60)$ & $140.70(15.45)$ & 126 \\
Heterogeneous treatment & $127.12(13.13)$ & $133.31(14.38)$ & $138.67(15.50)$ & $140.17(17.06)$ & 126 \\
No Identity treatment & $130.11(14.41)$ & $135.62(13.63)$ & $141.31(13.95)$ & $142.04(14.05)$ & 72 \\
\hline Least performers (excl. & & & & \\
No Feedback treatment) & & & & \\
$\quad$ - Not exposed & $116.22(13.51)$ & $123.80(11.46)$ & $126.58(11.41)$ & $130.54(12.16)$ & 81 \\
$\quad$ - Exposed & $121.25(13.06)$ & $123.47(16.89)$ & $127.39(18.93)$ & $127.71(22.97)$ & 51 \\
\hline
\end{tabular}

Note: N: number of subjects. Standard deviations are in parentheses.

Table 5. Determinants of the evolution of performance between part 3 and part 4, depending on rank in part 3

\begin{tabular}{|c|c|c|c|}
\hline $\begin{array}{l}\text { Dependent variable: } \\
\text { Performance in part } 4 \\
\text { - Performance in part } 3\end{array}$ & Triad & $\begin{array}{c}\text { Rank } 1 \text { or } 2 \\
\text { in part } 3 \\
(2)\end{array}$ & $\begin{array}{c}\text { Rank } 3 \\
\text { in part } 3 \\
(3)\end{array}$ \\
\hline No Feedback treatment & Ref. & Ref. & Ref. \\
\hline Minimal Identity treatment & $5.395(5.015)$ & $1.794(2.346)$ & $1.934(3.417)$ \\
\hline Homogenous treatment & $-1.584(4.715)$ & $0.182(2.077)$ & $-2.364(3.246)$ \\
\hline Heterogeneous treatment & $1.336(4.731)$ & $0.961(1.922)$ & $-1.178(3.150)$ \\
\hline No Identity treatment & $0.679(5.166)$ & $1.260(2.249)$ & $-2.364(3.411)$ \\
\hline Chose to pay the fee & - & $-0.512(1.220)$ & $1.611(1.583)$ \\
\hline Exposure of rank 3 in part 3 & $-4.831 * *(2.254)$ & $-0.856(1.253)$ & $-3.123 * *(1.472)$ \\
\hline Demographic variables & - & Yes & Yes \\
\hline Constant & $4.750(4.281)$ & $2.122(5.007)$ & $5.509(7.183)$ \\
\hline $\mathrm{N}$ & 140 & 278 & 140 \\
\hline Prob $>F$ & 0.092 & 0.735 & 0.039 \\
\hline $\mathrm{R}^{2}$ & 0.068 & 0.023 & 0.124 \\
\hline
\end{tabular}

Note: Table 5 reports OLS models. In model (2), standard errors are clustered at the triad level. In model (2), two observations are missing because the level of wealth has not been recorded. ** indicate significance at the 5\% level. 


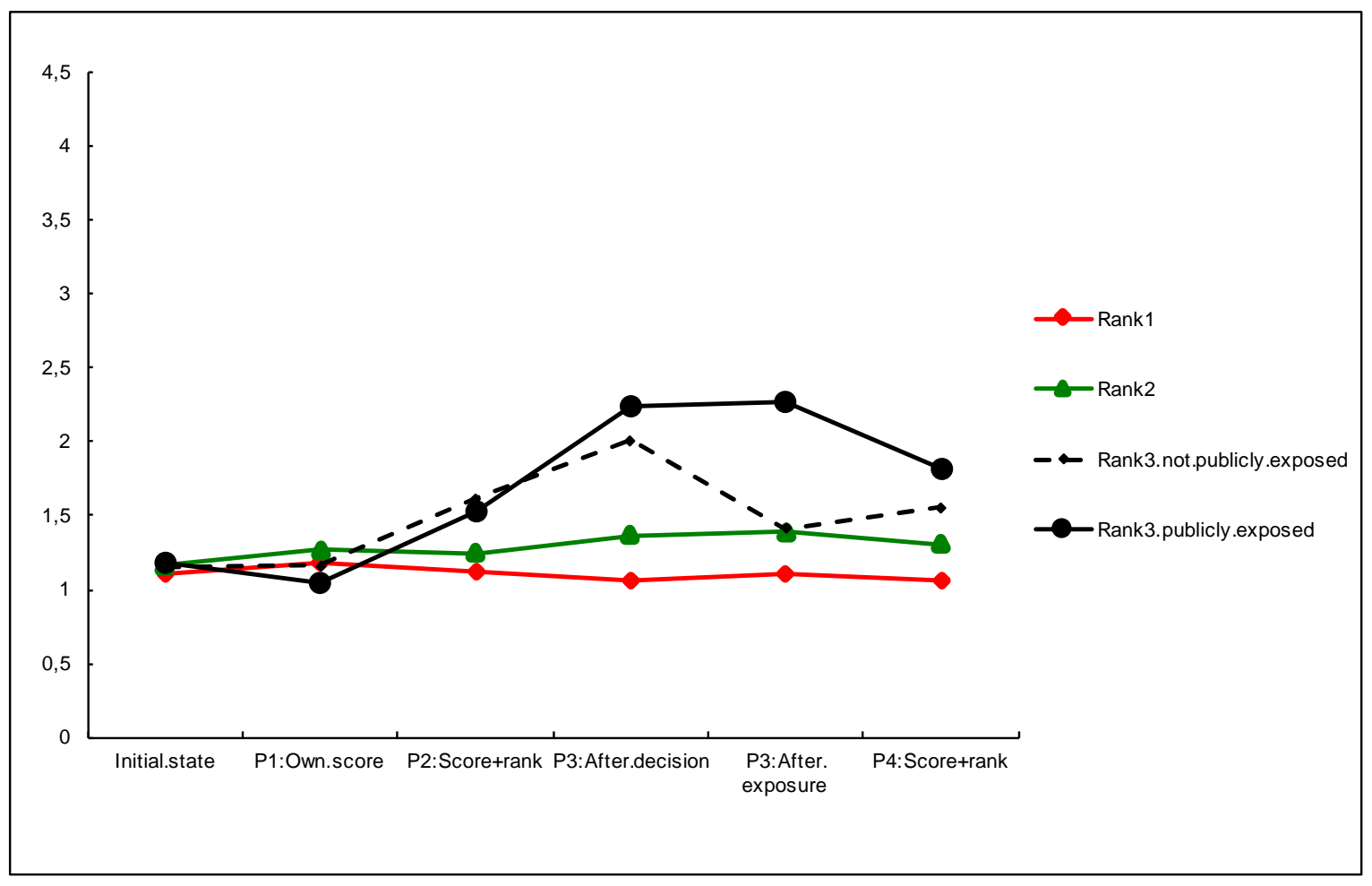

Figure 1. Shame intensity, by performance rank, in all treatments $(\mathrm{N}=396)$

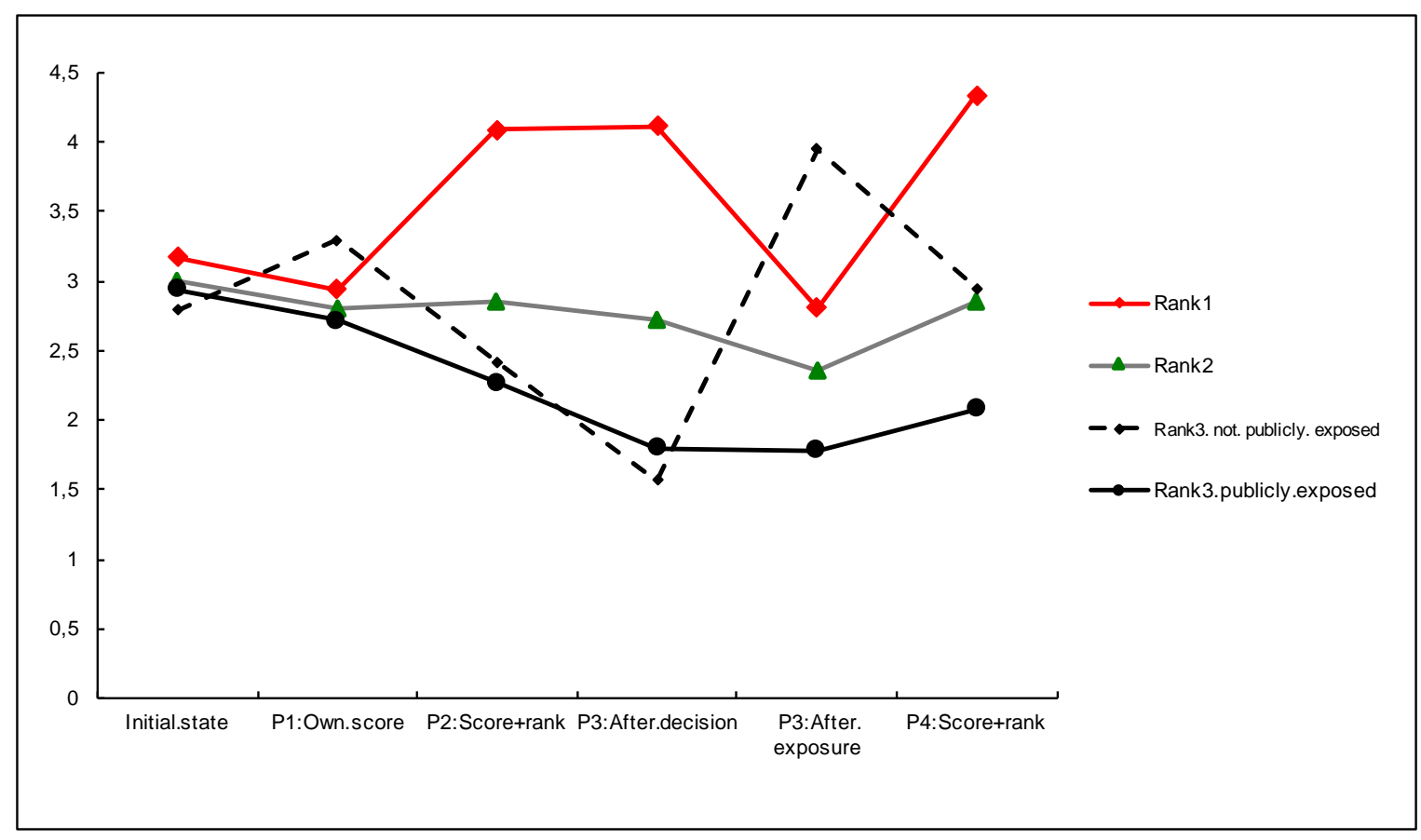

Figure 2. Happiness intensity, by performance rank, in all treatments $(\mathrm{N}=396)$ 


\section{ON LINE APPENDIX 1 - Instructions for the Minimal Identity treatment (translated into Chinese)}

We thank you for participating in this experiment in decision-making. It is forbidden to talk to the other participants throughout the session.

We will first ask you to describe the feelings you experience right now. Your screen will display a series of 10 statements. For each statement, we ask you to choose a number between 1 and 7 (included) to describe how you feel right now.

The choice of a higher number indicates that you experience this feeling with a higher intensity. For example, choosing number 1 indicates that you do not experience this feeling at all. At the opposite, choosing number 7 indicates that you experience this feeling with a very high intensity. Intermediate values are indicated by the choice of intermediate numbers.

Do not spend too much time on any one statement; simply choose the number which seems to describe your present feelings best.

The 10 proposed statements are the following:

- I feel angry

- I feel contempt

- I feel ashamed

- I feel envious

- I feel sad

- I feel happy

- I feel guilty

- I feel grateful

- I feel fearful

- $\quad$ I feel relieved

\section{Instructions (cont'd) (distributed after completion of the initial elicitation of feelings)}

The remaining of this session consists of several parts. You will receive the instructions for each part after completing the previous part.

During these parts, the amount of money you will earn may depend upon your actions and the actions of the other participants you will interact with. Throughout the session, your earnings will be given in ECU (Experimental Currency Units). At the end of the session, your total earnings in ECU will be the sum of your payoffs earned in each part. These earnings will be converted into RMB at the rate:

$$
10 \mathrm{ECU}=1 \mathrm{RMB}
$$

Your earnings will be paid to you in cash and in private at the end of the session.

\section{Part 1}

At the beginning of this part and throughout the session, the participants are grouped into triads. The composition of your triad will remain constant throughout the session. You will not be informed on the identity of the two other members of your triad.

During this part, we ask you to perform a task on your computer during 4 minutes. This task consists of moving apples into a basket with your mouse.

Each apple moved into the basket will increase your score by one unit. Your current score (i.e. the current number of 
apples in the basket) as well as the remaining time will be displayed on your screen continuously.

Once the 4 minutes have elapsed, your screen will remind you your score in this part. You will not be informed of the scores of your two other triad members.

You will be paid 100 ECU in this part.

Before starting performing the task, you will be allowed to practice this task during 2 minutes. The performance in this practice period will not be taken into account in your score of this part.

At the end of this part, a questionnaire about your current feelings will be displayed on your computer screen. To fill out this questionnaire, the rules are the same as previously.

If you have any question regarding these instructions, please raise your hand. We will answer to your questions privately.

\section{Part 2 (distributed after completion of the previous part)}

In this part, the composition of your triad is the same as in the previous part.

We ask you to perform the same task on your computer as previously during 4 minutes. You will be paid 100 ECU in this part.

The difference with the previous part is that once the 4 minutes have elapsed, your screen will display your score and your rank within the triad in this part.

The rank 1 will be assigned to the triad member who has performed the highest score.

The rank 3 will be assigned to the member who has performed the lowest score.

The rank 2 will be assigned to the member who has performed the intermediate score.

In case of ties between two or three triad members, the ranks will be assigned randomly between the ties.

You will be informed of the score and the rank of each of your two other triad members.

Last, a questionnaire about your current feelings will be displayed on your computer screen.

If you have any question regarding these instructions, please raise your hand. We will answer to your questions privately.

\section{Part 3 (distributed after completion of the previous part)}

In this part, the composition of your triad is the same as in the previous parts. We ask you to perform the same task on your computer as previously during 4 minutes. You will be paid 100 ECU in this part. Once the 4 minutes have elapsed, your screen will display your score and your rank within the triad, as well as the score and the rank of each of the two other triad members.

A difference with the previous part lies in the fact that the participants who have got rank 3 (the lowest score) in each triad will be requested to stand up, to move forward to the front of the room, one by one, before being sent back to their seat.

However, you will be able to modify the regular process by your decisions. Indeed, after being informed on the assignment of ranks and before participants with rank 3 (the lowest score) are called to the front of the room, you will decide whether you let the regular process apply or whether you prefer that the member of your triad who has got rank 3 is not called to the front of the room.

- If you are willing that the regular process applies, press «VALIDATE». 
- If you are not willing that the member of your triad with rank 3 is called to the front of the room, press «NO STAND $\mathrm{UP} »$ and validate your decision.

If at least two members of the triad have pressed « NO STAND UP », the member of your triad who has got rank 3 will not be called and will not be identified in the room. In this case, the members who have chosen «NO STAND UP» will pay a cost of $10 \mathrm{ECU}$ each that will be deducted from their payoff in this part.

In all the other cases, the regular process applies. The member of your triad with rank 3 will be called to the front of the room. Payoffs will not be modified.

You will be informed of the decision of each of your triad members, together with their score and their rank.

A questionnaire about your current feelings will be displayed on your computer screen both after you have made your decision and after the participants with rank 3 have come back to their seat.

If you have any question regarding these instructions, please raise your hand. We will answer to your questions privately.

\section{Part 4 (distributed after completion of the previous part)}

The rules that apply in this part are the same as in Part 2. We ask you to perform the same task as previously during 4 minutes. You will be paid $100 \mathrm{ECU}$ in this part. Once the 4 minutes have elapsed, your screen will display your score and your rank within the triad in this part. You will be also informed of the score and the rank of each of the two other triad members. Then, a questionnaire about your current feelings will be displayed on your computer screen.

Finally, you will be requested to fill out a post-experimental questionnaire and you will be invited to proceed to the payment room. 


\section{ON LINE APPENDIX 2 - Instructions for the Homogenous and Heterogeneous treatments (translated into Chinese)}

We thank you for participating in this experiment in decision-making. It is forbidden to talk to the other participants throughout the session.

We will first ask you to describe the feelings you experience right now. Your screen will display a series of 10 statements. For each statement, we ask you to choose one number between 1 and 7 (included) to describe how you feel right now.

The choice of a higher number indicates that you experience this feeling with a higher intensity. For example, choosing number 1 indicates that you do not experience this feeling at all. At the opposite, choosing number 7 indicates that you experience this feeling with a very high intensity. Intermediate values are indicated by the choice of intermediate numbers.

Do not spend too much time on any one statement; simply choose the number which seems to describe your present feelings best.

The 10 proposed statements are the following:

$\begin{array}{ll}\text { - } & \text { I feel angry } \\ \text { - } & \text { I feel contempt } \\ \text { - } & \text { I feel ashamed } \\ \text { - } & \text { I feel envious } \\ \text { - } & \text { I feel sad } \\ \text { - } & \text { I feel happy } \\ \text { - } & \text { I feel guilty } \\ \text { - } & \text { I feel grateful } \\ \text { - } & \text { I feel fearful } \\ \text { - } & \text { I feel relieved }\end{array}$

\section{Instructions (cont'd) (distributed after completion of the initial elicitation of feelings)}

The remaining of this session consists of several parts. You will receive the instructions for each part after completing the previous part.

During these parts, the amount of money you will earn may depend upon your actions and the actions of the other participants you will interact with. Throughout the session, your earnings will be given in ECU (Experimental Currency Units). At the end of the session, your total earnings in ECU will be the sum of your payoffs earned in each part. These earnings will be converted into RMB at the rate:

$$
10 \mathrm{ECU}=1 \mathrm{RMB}
$$

Your earnings will be paid to you in cash and in private at the end of the session.

\section{Preliminary part}

At the beginning of this part and for the remainder of the session, the participants will be classified into one of two groups of equal size. Each group is called after the name of an artist: Klee or Kandinsky. You will be randomly assigned to one of these two groups, either the Klee group, or the Kandinsky group. You will belong to the same group throughout the session.

After informing you about your group membership, your screen will display five pairs of paintings. In each pair, a painting has been made by Klee, the other one by Kandinsky. Your screen will indicate which artist has painted each painting. Each pair of paintings will be displayed during 20 seconds.

After observing these five pairs of paintings, your screen will successively display two more paintings and you will be asked to select the artist who you think made each painting, respectively (Klee or Kandinsky). Each correct answer will pay you 50 ECU. 
You may get help from or help other members in your own group while answering the two questions successively. Indeed, before submitting each of your answers, you can use a group chat program during 3 minutes to get help from or offer help to other members in your own group.

Except for the following restrictions, you can type whatever you want in the lower box of the chat program:

- Please do not identify yourself or send any information that could be used to identify you (age, gender, school, ...),

- Please refrain from using obscene or offensive language.

To send a message to your group members, simply press the button.

Your messages will be shared only with the members of your own group. You will not be able to see the messages exchanged among the other group. People in the other group will not see the messages from your own group either.

Once the communication time has elapsed, you will be requested to enter your answer about the artist you think has made the painting displayed on your screen (Klee or Kandinsky).

You will be informed at the end of the session whether your answers were correct or not.

Once you have submitted your two answers, a questionnaire on your current feelings will be displayed on your computer screen. To fill out this questionnaire, the rules are the same as previously.

Please read these instructions again. If you have any question on these instructions, please raise your hand. We will answer your questions privately.

\section{Part 1}

At the beginning of this part and throughout the session, the participants are grouped into triads. The composition of your triad will remain constant throughout the session. You will not be informed on the identity of the other members of your triad. In contrast, you will be informed of the group of each of the two other triad members (Klee or Kandinsky); similarly, your triad members will learn your group.

During this part, we ask you to perform a task on your computer during 4 minutes. This task consists of moving apples into a basket with your mouse.

Each apple moved into the basket will increase your score by one unit. Your current score (i.e. the current number of apples in the basket) as well as the remaining time will be displayed on your screen continuously.

Once the 4 minutes have elapsed, your screen will remind you your score in this part. You will not be informed of the scores of your two other triad members.

You will be paid $100 \mathrm{ECU}$ in this part.

Before starting performing the task, you will be allowed to practice this task during 2 minutes. The performance in this practice period will not be taken into account in your score of this part.

At the end of this part, a questionnaire about your current feelings will be displayed on your computer screen.

If you have any question regarding these instructions, please raise your hand. We will answer to your questions privately.

\section{Part 2 (distributed after completion of the previous part)}

In this part, the composition of your triad is the same as in the previous part. We ask you to perform the same task on your computer as previously during 4 minutes. You will be paid $100 \mathrm{ECU}$ in this part.

The difference with the previous part is that once the 4 minutes have elapsed, your screen will display your score and your rank within the triad in this part. 
The rank 1 will be assigned to the triad member who has performed the highest score.

The rank 3 will be assigned to the member who has performed the lowest score.

The rank 2 will be assigned to the member who has performed the intermediate score.

In case of ties between two or three triad members, the ranks will be assigned randomly between the ties.

You will be informed of the group (Klee or Kandinsky), the score and the rank of each of your two other triad members. Last, a questionnaire about your current feelings will be displayed on your computer screen.

If you have any question regarding these instructions, please raise your hand. We will answer to your questions privately.

\section{Part 3 (distributed after completion of the previous part)}

In this part, the composition of your triad is the same as in the previous parts. We ask you to perform the same task on your computer as previously during 4 minutes. You will be paid 100 ECU in this part.

Once the 4 minutes have elapsed, your screen will display your score and your rank within the triad, as well as the group (Klee or Kandinsky), the score and the rank of each of the two other triad members.

A difference with the previous part lies in the fact that the participants who have got rank 3 (the lowest score) in each triad will be requested to stand up, to move forward to the front of the room, one by one, and then to give the name of their group (Klee or Kandinsky) aloud before being sent back to their seat.

However, you will be able to modify the regular process by your decisions. Indeed, after being informed on the assignment of ranks and before participants with rank 3 (the lowest score) are called to the front of the room, you will decide whether you let the regular process apply or whether you prefer that the member of your triad who has got rank 3 is not called to the front of the room.

- If you are willing that the regular process applies, press «VALIDATE».

- If you are not willing that the member of your triad with rank 3 is called to the front of the room, press «NO STAND UP » and validate your decision.

If at least two members of the triad have pressed «NO STAND UP », the member of your triad who has got rank 3 will not be called and will not be identified in the room. In this case, the members who have chosen « NO STAND UP » will pay a cost of $10 \mathrm{ECU}$ each that will be deducted from their payoff in this part.

In all the other cases, the regular process applies. The member of your triad with rank 3 will be called to the front of the room, will give the name of his group aloud and will come back to his seat. Payoffs will not be modified.

You will be informed of the decision of each of your triad members, together with their group, their score and their rank.

A questionnaire about your feelings will be displayed on your computer screen both after you have made your decision and after the participants with rank 3 have come back to their seat.

If you have any question regarding these instructions, please raise your hand. We will answer to your questions privately.

\section{Part 4 (distributed after completion of the previous part)}

The rules that apply in this part are the same as in part 2. We ask you to perform the same task as previously during 4 minutes. You will be paid $100 \mathrm{ECU}$ in this part.

Once the 4 minutes have elapsed, your screen will display your score and your rank within the triad in this part. You 
will be also informed of the group (Klee or Kandinsky), the score and the rank of each of the two other triad members. Then, a questionnaire about your current feelings will be displayed on your computer screen.

Finally, you will be requested to fill out a post-experimental questionnaire and you will be invited to proceed to the payment room. 
ON LINE APPENDIX 3. Table A1. Mean performance at the triad level, by part and by treatment

\begin{tabular}{|c|c|c|c|c|c|}
\hline & Part 1 & Part 2 & Part 3 & Part 4 & $\mathrm{~N}$ \\
\hline \multicolumn{6}{|c|}{ Minimal Identity treatment } \\
\hline - Not exposed & $378.20(16.79)$ & $398.40(20.54)$ & $410.07(20.34)$ & 421.07 (17.67) & 15 \\
\hline - Exposed & $386.78(18.89)$ & $396.44(35.22)$ & $405.22(37.74)$ & $409.11(39.11)$ & 9 \\
\hline \multicolumn{6}{|c|}{ Homogenous treatment } \\
\hline - Not exposed & $377.87(28.02)$ & $404.47(28.63)$ & $419.43(26.72)$ & $420.23(29.47)$ & 30 \\
\hline - Exposed & $384.75(21.04)$ & $409.25(22.70)$ & $422.50(20.77)$ & $426.75(16.68)$ & 12 \\
\hline \multicolumn{6}{|c|}{ Heterogeneous treatment } \\
\hline - Not exposed & $376.46(18.10)$ & $399.39(18.78)$ & $414.29(22.22)$ & $421.46(20.74)$ & 28 \\
\hline - Exposed & $391.14(26.88)$ & $401.00(29.83)$ & $419.50(30.51)$ & 418.57 (42.19) & 14 \\
\hline \multicolumn{6}{|l|}{ No Identity treatment } \\
\hline - Not exposed & $383.75(26.12)$ & $404.75(28.60)$ & $416.38(29.89)$ & $425.25(21.21)$ & 8 \\
\hline - Exposed & $393.63(24.36)$ & $407.94(22.06)$ & $427.69(24.61)$ & $426.56(20.23)$ & 16 \\
\hline
\end{tabular}

Note: Table A1 reports the mean total performance at the triad level in each part, depending on the treatment and on whether the least performer in the triad has or not been exposed in part 3. $\mathrm{N}$ indicates the number of triads in each condition. Standard deviations are in parentheses. In the No Identity treatment, mean performance at the triad level across parts is based on the triad as formed in part 3 .

P-values from Wilcoxon tests comparing performance at the triad level between part 3 and part 4, by treatment

\begin{tabular}{lcc}
\hline Treatments & $\begin{array}{c}\text { In case of exposure } \\
\text { of the least performer }\end{array}$ & $\begin{array}{c}\text { In case of no exposure } \\
\text { of the least performer }\end{array}$ \\
\hline Minimal Identity & 0.659 & 0.178 \\
Homogenous & 0.644 & 0.959 \\
Heterogeneous & 1.000 & 0.258 \\
No Identity & 0.925 & 0.495 \\
No Feedback & - & 0.528 \\
\hline
\end{tabular}

Note: In the No Identity treatment, triads are reformed randomly at the beginning of each part; for the test, however, we compare performance in part 3 and part 4 at the triad level as composed in part 3 . In the No Feedback treatment, subjects are not matched in triads; therefore, in this treatment the test is conducted at the individual level. 